\title{
Sensitivity to light sterile neutrino mixing parameters with KM3NeT/ORCA
}

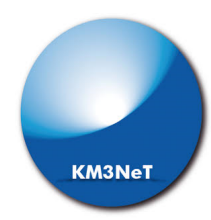

\section{The KM3NeT collaboration}

S. Aiello, ${ }^{a}$ A. Albert, ${ }^{b c}, b$ M. Alshamsi, ${ }^{c}$ S. Alves Garre, ${ }^{d}$ Z. Aly, ${ }^{e}$ A. Ambrosone, ${ }^{f, g}$ F. Ameli, ${ }^{h}$ M. Andre, ${ }^{i}$ G. Androulakis, ${ }^{j, 1}$ M. Anghinolfi, ${ }^{k}$ M. Anguita, ${ }^{l}$ G. Anton, ${ }^{m}$ M. Ardid, ${ }^{n}$ S. Ardid, ${ }^{n}$ J. Aublin, ${ }^{c}$ C. Bagatelas, ${ }^{j}$ B. Baret,,${ }^{c}$ S. Basegmez du Pree, ${ }^{o}$ M. Bendahman, ${ }^{c, p}$ F. Benfenati, ${ }^{q, r}$ E. Berbee, ${ }^{o}$ A.M. van den Berg, ${ }^{s}$ V. Bertin, ${ }^{e}$ S. Biagi, ${ }^{t}$ M. Bissinger, ${ }^{m}$ M. Boettcher, ${ }^{u}$ M. Bou Cabo, ${ }^{v}$ J. Boumaaza, ${ }^{p}$ M. Bouta, ${ }^{w}$ M. Bouwhuis, ${ }^{o}$ C. Bozza, ${ }^{x}$ H. Brânzaș, ${ }^{y}$ F. Bretaudeau, ${ }^{z}$ R. Bruijn, ${ }^{o, a}{ }^{a}$ J. Brunner, ${ }^{e}$ R. Bruno, ${ }^{a}$ E. Buis, ${ }^{a b}$ R. Buompane, ${ }^{f, a c}$ J. Busto, ${ }^{e}$ B. Caiffi, ${ }^{k}$ D. Calvo, ${ }^{d}$ S. Campion, ${ }^{a e, h}$ A. Capone, ${ }^{a e, h}$ V. Carretero, ${ }^{d}$ P. Castaldi, ${ }^{q, a f}$ S. Celli, ${ }^{a e, h}$ M. Chabab, ${ }^{a g}$ N. Chau, ${ }^{c}$ A. Chen, ${ }^{a h}$ S. Cherubini, ${ }^{t a i}$ V. Chiarella, ${ }^{a j}$ T. Chiarusi, ${ }^{q}$ M. Circella, ${ }^{a k}$ R. Cocimano, ${ }^{t}$ J.A.B. Coelho, ${ }^{c, 2}$ A. Coleiro, ${ }^{c}$ M. Colomer Molla, ${ }^{c, d}$ R. Coniglione, ${ }^{t}$ P. Coyle, ${ }^{e}$ A. Creusot, ${ }^{c}$ A. Cruz, ${ }^{a l}$ G. Cuttone, ${ }^{t}$ R. Dallier, ${ }^{z}$ B. De Martino, ${ }^{e}$ M. De Palma, ${ }^{a k, a m}$ I. Di Palma, ${ }^{a e, h}$ A.F. Díaz, ${ }^{l}$ D. Diego-Tortosa, ${ }^{n}$ C. Distefano, ${ }^{t}$ A. Domi, ${ }^{k, a n, 2}$ C. Donzaud, ${ }^{c}$ D. Dornic, ${ }^{e}$ M. Dörr, ${ }^{a o}$ D. Drouhin, $, b c, b$ T. Eberl, ${ }^{m}$ A. Eddyamoui, ${ }^{p}$ T. van Eeden, ${ }^{o}$ D. van Eijk, ${ }^{o}$ I. El Bojaddaini, ${ }^{w}$ D. Elsaesser, ${ }^{a o}$ A. Enzenhöfer, ${ }^{e}$ V. Espinosa, ${ }^{n}$ P. Fermani, ${ }^{a e, h}$ G. Ferrara, ${ }^{t, a i}$ M.D. Filipović, ${ }^{a p}$ F. Filippini, ${ }^{q, r}$ L.A. Fusco, ${ }^{e}$ T. Gal, ${ }^{m}$ J. García Méndez, ${ }^{n}$

A. Garcia Soto, ${ }^{o}$ F. Garufi, ${ }^{f, g}$ Y. Gatelet, ${ }^{c}$ N. Geißelbrecht, ${ }^{m}$ L. Gialanella, ${ }^{f, a c}$

E. Giorgio, ${ }^{t}$ S.R. Gozzini, ${ }^{a e, h}$ R. Gracia, ${ }^{o}$ K. Graf, ${ }^{m}$ G. Grella, ${ }^{a q}$ D. Guderian, ${ }^{b d}$

C. Guidi, ${ }^{k, a n}$ M. Gutiérrez, ${ }^{a r}$ J. Haefner, ${ }^{m}$ S. Hallmann, ${ }^{m}$ H. Hamdaoui, ${ }^{p}$

H. van Haren, ${ }^{a s}$ A. Heijboer, ${ }^{o}$ A. Hekalo, ${ }^{o o}$ L. Hennig, ${ }^{m}$ J.J. Hernández-Rey, ${ }^{d}$ J. Hofestädt, ${ }^{m}$ F. Huang, ${ }^{e}$ W. Idrissi Ibnsalih, ${ }^{f, a c}$ G. Illuminati, ${ }^{q, c}$ C.W. James, ${ }^{a l}$ M. de Jong, ${ }^{o}$ P. de Jong, ${ }^{o, a a}$ B.J. Jung, ${ }^{o}$ M. Kadler, ${ }^{o o}$ P. Kalaczyński, ${ }^{a t}$ O. Kalekin, ${ }^{m}$

${ }^{1}$ Deceased.

${ }^{2}$ Corresponding author. 
U.F. Katz, ${ }^{m}$ N.R. Khan Chowdhury, ${ }^{d}$ G. Kistauri, ${ }^{a u}$ F. van der Knaap, ${ }^{a b}$ P. Kooijman, ${ }^{a, b e}$ A. Kouchner, ${ }^{c, a v}$ V. Kulikovskiy, ${ }^{k}$ R. Lahmann, ${ }^{m}$ M. Lamoureux, ${ }^{c}$

G. Lara, ${ }^{v}$ G. Larosa, ${ }^{t}$ C. Lastoria, ${ }^{e}$ R. Le Breton, ${ }^{c}$ S. Le Stum, ${ }^{e}$ O. Leonardi, ${ }^{t}$

F. Leone, ${ }^{t, a i}$ E. Leonora, ${ }^{a}$ N. Lessing, ${ }^{m}$ G. Levi, ${ }^{q, r}$ M. Lincetto, ${ }^{e}$ M. Lindsey Clark, ${ }^{c}$

T. Lipreau, ${ }^{z}$ F. Longhitano, ${ }^{a}$ D. Lopez-Coto, ${ }^{a r}$ A. Lygda, ${ }^{j}$ L. Maderer, ${ }^{c}$ J. Mańczak, ${ }^{d}$

K. Mannheim, ${ }^{a o}$ A. Margiotta, ${ }^{q, r}$ A. Marinelli, ${ }^{f}$ C. Markou, ${ }^{j}$ L. Martin, ${ }^{z}$

J.A. Martínez-Mora, ${ }^{n}$ A. Martini, ${ }^{a j}$ F. Marzaioli, ${ }^{f, a c}$ S. Mastroianni, ${ }^{f}$ K.W. Melis, ${ }^{o}$

G. Miele, ${ }^{f, g}$ P. Migliozzi, ${ }^{f}$ E. Migneco, ${ }^{t}$ P. Mijakowski, ${ }^{a t}$ L.S. Miranda, ${ }^{a w}$

C.M. Mollo, ${ }^{f}$ M. Moser, ${ }^{m}$ A. Moussa, ${ }^{w}$ R. Muller, ${ }^{o}$ M. Musumeci, ${ }^{t}$ L. Nauta, ${ }^{o}$

S. Navas, ${ }^{a r}$ C.A. Nicolau, ${ }^{h}$ B. Nkosi, ${ }^{a h}$ B. Ó Fearraigh, ${ }^{o, a a}$ M. O'Sullivan, ${ }^{a l}$

M. Organokov, ${ }^{b}$ A. Orlando, ${ }^{t}$ J. Palacios González, ${ }^{d}$ G. Papalashvili, ${ }^{a u}$ R. Papaleo, ${ }^{t}$

C. Pastore, ${ }^{a k}$ A.M. Păun, ${ }^{y}$ G.E. Păvălaș, ${ }^{y}$ C. Pellegrino, ${ }^{r, b f}$ S. Peña Martínez, ${ }^{e}$

M. Perrin-Terrin, ${ }^{e}$ V. Pestel, ${ }^{o}$ P. Piattelli, ${ }^{t}$ C. Pieterse, ${ }^{d}$ O. Pisanti, ${ }^{f, g}$ C. Poirè, ${ }^{n}$

V. Pontoriere, ${ }^{f}$ V. Popa, ${ }^{y}$ T. Pradier, ${ }^{b}$ I. Probst, ${ }^{m}$ G. Pühlhofer, ${ }^{a x}$ S. Pulvirenti, ${ }^{t}$

N. Randazzo, ${ }^{a}$ S. Razzaque, ${ }^{a w}$ D. Real, ${ }^{d}$ S. Reck, ${ }^{m}$ G. Riccobene, ${ }^{t}$ A. Romanov, ${ }^{k, a n}$

A. Rovelli, ${ }^{t}$ F. Salesa Greus, ${ }^{d}$ D.F.E. Samtleben, ${ }^{o, a y}$ A. Sánchez Losa, ${ }^{a k}$

M. Sanguineti, ${ }^{k, a n}$ A. Santangelo, ${ }^{a x}$ D. Santonocito, ${ }^{t}$ P. Sapienza, ${ }^{t}$ J. Schnabel, ${ }^{m}$

M.F. Schneider, ${ }^{m}$ J. Schumann, ${ }^{m}$ H.M. Schutte, ${ }^{u}$ J. Seneca, ${ }^{o}$ I. Sgura, ${ }^{a k}$

R. Shanidze, ${ }^{a u}$ A. Sharma, ${ }^{a z}$ A. Sinopoulou, ${ }^{j}$ B. Spisso, ${ }^{a q, f}$ M. Spurio, ${ }^{q, r}$

D. Stavropoulos, ${ }^{j}$ S.M. Stellacci, ${ }^{a q, f}$ M. Taiuti, ${ }^{k, a n}$ F. Tatone, ${ }^{a k}$ Y. Tayalati, ${ }^{p}$

T. Thakore,,$^{d, 2,3} \mathbf{H}$. Thiersen, ${ }^{u} \mathbf{S}$. Tingay, ${ }^{a l} \mathbf{S}$. Tsagkli, ${ }^{j}$ V. Tsourapis, ${ }^{j}$

E. Tzamariudaki, ${ }^{j}$ D. Tzanetatos, ${ }^{j}$ V. Van Elewyck, ${ }^{c, a v}$ G. Vasileiadis, ${ }^{b a}$ F. Versari, ${ }^{q, r}$

D. Vivolo, ${ }^{f, a c}$ G. de Wasseige, ${ }^{c}$ J. Wilms, ${ }^{b b}$ R. Wojaczyński, ${ }^{a t}$ E. de Wolf, ${ }^{o, a a}$

T. Yousfi, ${ }^{w}$ S. Zavatarelli, ${ }^{k}$ A. Zegarelli, ${ }^{a e, h}$ D. Zito, ${ }^{t}$ J.D. Zornoza, ${ }^{d}$ J. Zúñiga ${ }^{d}$ and

N. Zywucka ${ }^{u}$

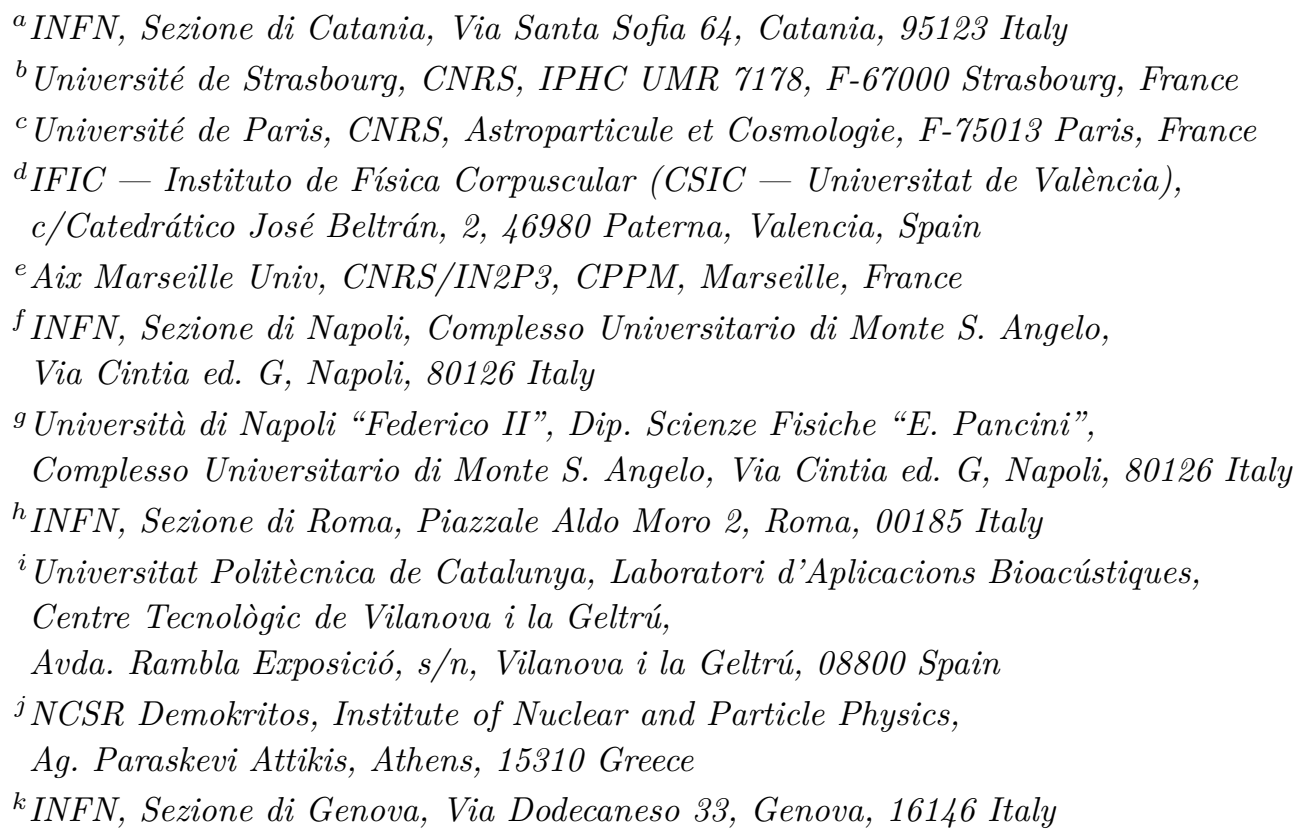

${ }^{3}$ Presently at the University of Cincinnati, Ohio, United States. 
${ }^{l}$ University of Granada, Dept. of Computer Architecture and Technology/CITIC, 18071 Granada, Spain

${ }^{m}$ Friedrich-Alexander-Universität Erlangen-Nürnberg, Erlangen Centre for Astroparticle Physics, Erwin-Rommel-Straße 1, 91058 Erlangen, Germany

${ }^{n}$ Universitat Politècnica de València, Instituto de Investigación para la Gestión Integrada de las Zonas Costeras, C/ Paranimf, 1, Gandia, 46730 Spain

${ }^{\circ}$ Nikhef, National Institute for Subatomic Physics, PO Box 41882, Amsterdam, 1009 DB Netherlands

${ }^{p}$ University Mohammed $V$ in Rabat, Faculty of Sciences, 4 av. Ibn Battouta, B.P. 1014, R.P. 10000 Rabat, Morocco

${ }^{q}$ INFN, Sezione di Bologna, v.le C. Berti-Pichat, 6/2, Bologna, 40127 Italy

${ }^{r}$ Università di Bologna, Dipartimento di Fisica e Astronomia, v.le C. Berti-Pichat, 6/2, Bologna, 40127 Italy

${ }^{s}$ KVI-CART University of Groningen, Groningen, the Netherlands

${ }^{t}$ INFN, Laboratori Nazionali del Sud, Via S. Sofia 62, Catania, 95123 Italy

${ }^{u}$ North-West University, Centre for Space Research,

Private Bag X6001, Potchefstroom, 2520 South Africa

${ }^{v}$ Instituto Español de Oceanografía, Unidad Mixta IEO-UPV,

C/ Paranimf, 1, Gandia, 46730 Spain

${ }^{w}$ University Mohammed I, Faculty of Sciences, BV Mohammed VI,

B.P. 717, R.P. 60000 Oujda, Morocco

${ }^{x}$ Università di Salerno e INFN Gruppo Collegato di Salerno, Dipartimento di Matematica, Via Giovanni Paolo II 132, Fisciano, 84084 Italy

${ }^{y}$ ISS, Atomistilor 409, Măgurele, RO-07r125 Romania

${ }^{z}$ Subatech, IMT Atlantique, IN2P3-CNRS, Université de Nantes, 4 rue Alfred Kastler — La Chantrerie, Nantes, BP 20722 44307 France

${ }^{a a}$ University of Amsterdam, Institute of Physics/IHEF,

PO Box 94216, Amsterdam, 1090 GE Netherlands

${ }^{a b}$ TNO, Technical Sciences, PO Box 155, Delft, 2600 AD Netherlands

${ }^{a c}$ Università degli Studi della Campania "Luigi Vanvitelli", Dipartimento di Matematica e Fisica, viale Lincoln 5, Caserta, 81100 Italy

${ }^{a e}$ Università La Sapienza, Dipartimento di Fisica, Piazzale Aldo Moro 2, Roma, 00185 Italy

${ }^{a f}$ Università di Bologna, Dipartimento di Ingegneria dell'Energia Elettrica e dell'Informazione

"Guglielmo Marconi", Via dell'Università 50, Cesena, 47521 Italia

${ }^{a g}$ Cadi Ayyad University, Physics Department, Faculty of Science Semlalia,

Av. My Abdellah, P.O.B. 2390, Marrakech, 40000 Morocco

${ }^{a h}$ University of the Witwatersrand, School of Physics,

Private Bag 3, Johannesburg, Wits 2050 South Africa

${ }^{a i}$ Università di Catania, Dipartimento di Fisica e Astronomia "Ettore Majorana", Via Santa Sofia 64, Catania, 95123 Italy

${ }^{a j}$ INFN, LNF, Via Enrico Fermi, 40, Frascati, 00044 Italy

${ }^{a k}$ INFN, Sezione di Bari, via Orabona, 4, Bari, 70125 Italy

${ }^{a l}$ International Centre for Radio Astronomy Research, Curtin University, Bentley, WA 6102, Australia

${ }^{\text {am }}$ University of Bari, Via Amendola 173, Bari, 70126 Italy 
${ }^{a n}$ Università di Genova, Via Dodecaneso 33, Genova, 16146 Italy

ao University Würzburg, Emil-Fischer-Straße 31, Würzburg, 97074 Germany

${ }^{a p}$ Western Sydney University, School of Computing, Engineering and Mathematics,

Locked Bag 1797, Penrith, NSW 2751 Australia

aq Università di Salerno e INFN Gruppo Collegato di Salerno, Dipartimento di Fisica,

Via Giovanni Paolo II 132, Fisciano, 84084 Italy

${ }^{a r}$ University of Granada, Dpto. de Física Teórica y del Cosmos \& C.A.F.P.E., 18071 Granada, Spain

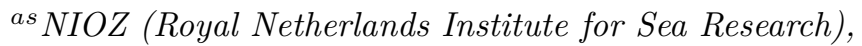
PO Box 59, Den Burg, Texel, 1790 AB, the Netherlands

${ }^{a t}$ National Centre for Nuclear Research, 02-093 Warsaw, Poland

${ }^{a u}$ Tbilisi State University, Department of Physics, 3, Chavchavadze Ave., Tbilisi, 0179 Georgia

${ }^{a v}$ Institut Universitaire de France, 1 rue Descartes, Paris, 75005 France

aw University of Johannesburg, Department Physics, PO Box 524, Auckland Park, 2006 South Africa

${ }^{a x}$ Eberhard Karls Universität Tübingen, Institut für Astronomie und Astrophysik,

Sand 1, Tübingen, 72076 Germany

${ }^{a y}$ Leiden University, Leiden Institute of Physics, PO Box 9504, Leiden, 2300 RA Netherlands

${ }^{a z}$ Università di Pisa, Dipartimento di Fisica, Largo Bruno Pontecorvo 3, Pisa, 56127 Italy

${ }^{b a}$ Laboratoire Univers et Particules de Montpellier,

Place Eugène Bataillon - CC 72, Montpellier Cédex 05, 34095 France

${ }^{b b}$ Friedrich-Alexander-Universität Erlangen-Nürnberg, Remeis Sternwarte, Sternwartstraße 7, 96049 Bamberg, Germany

${ }^{b c}$ Université de Strasbourg, Université de Haute Alsace, GRPHE, 34, Rue du Grillenbreit, Colmar, 68008 France

${ }^{b d}$ University of Münster, Institut für Kernphysik, Wilhelm-Klemm-Str. 9, Münster, 48149 Germany

${ }^{b e}$ Utrecht University, Department of Physics and Astronomy, PO Box 80000, Utrecht, 3508 TA Netherlands

${ }^{b f}$ INFN, CNAF, v.le C. Berti-Pichat, 6/2, Bologna, 40127 Italy

E-mail: jcoelho@apc.in2p3.fr, alba.domi@ge.infn.it, tarak.thakore@uc.edu, km3net-pc@km3net.de

ABSTRACT: KM3NeT/ORCA is a next-generation neutrino telescope optimised for atmospheric neutrino oscillations studies. In this paper, the sensitivity of ORCA to the presence of a light sterile neutrino in a $3+1$ model is presented. After three years of data taking, ORCA will be able to probe the active-sterile mixing angles $\theta_{14}, \theta_{24}, \theta_{34}$ and the effective angle $\theta_{\mu e}$, over a broad range of mass squared difference $\Delta m_{41}^{2} \sim\left[10^{-5}, 10\right] \mathrm{eV}^{2}$, allowing to test the eV-mass sterile neutrino hypothesis as the origin of short baseline anomalies, as well as probing the hypothesis of a very light sterile neutrino, not yet constrained by cosmology. ORCA will be able to explore a relevant fraction of the parameter space not yet reached by present measurements.

Keywords: Neutrino Detectors and Telescopes (experiments), Oscillation

ARXIV EPRINT: 2107.00344 


\section{Contents}

1 Introduction 1

2 The KM3NeT/ORCA detector 3

3 Theoretical background 4

3.1 Large $\left|\Delta m_{41}^{2}\right|$ limit 6

$\begin{array}{lll}3.1 .1 & \text { The ORCA low energy regime } & 7\end{array}$

3.1.2 The ORCA high energy regime 8

3.2 Finite $\left|\Delta m_{41}^{2}\right|$ regime $\quad 9$

4 Sterile neutrino analysis $\quad 9$

5 Sensitivity results 14

5.1 Sensitivity to $\theta_{24}-\theta_{34}$ in the large $\Delta m_{41}^{2}$ limit $\quad 14$

$\begin{array}{lll}5.2 & \text { Sensitivity to } \theta_{24} \text { for different } \Delta m_{41}^{2} \text { values } & 15\end{array}$

5.3 Sensitivity to $\theta_{14}$ for different $\Delta m_{41}^{2}$ values $\quad 16$

5.4 Sensitivity to $\left|U_{\mu e}\right|^{2}$ for different $\Delta m_{41}^{2}$ values $\quad 16$

$\begin{array}{lll}\text { 5.5 Sensitivity to } \theta_{34} \text { for different } \Delta m_{41}^{2} \text { values } & 17\end{array}$

6 Summary and conclusions $\quad 17$

\section{Introduction}

The study of neutrino oscillations has seen remarkable progress in the last three decades. An increasing number of solar, atmospheric and accelerator neutrino experiments have performed precision measurements of the neutrino oscillation parameters [1]. The experimental data is consistent with the three weakly-interacting neutrino picture (here referred to as the standard picture). Nevertheless, a number of questions remain unanswered, in particular what is the Neutrino Mass Ordering (NMO) and whether neutrino oscillations violate the CP symmetry. Upcoming experiments such as KM3NeT/ORCA [2], SBN [3], DUNE [4], JUNO [5], Hyper-K [6], IceCube/Gen2 [7] and INO [8] aim to resolve these questions over the next decades.

At the same time, several short baseline (SBL) neutrino experiments have reported anomalous experimental results which are inconsistent with the standard picture. A comprehensive review can be found in ref. [9]. Such results could be explained by assuming the existence of an additional neutrino (hereafter SBL neutrino). However, the Z-width measurement [10] has demonstrated that only three neutrinos can participate to weak interactions, for which they are referred as active neutrinos. Therefore, the SBL neutrino, 
not being able to participate to weak interactions, is called sterile. The SBL sterile neutrino should be light $\left(\Delta m_{41}^{2} \sim 1 \mathrm{eV}^{2}\right)$ and its presence affects the standard neutrino oscillation probabilities via its mixing with active neutrinos, in the so called $3+1$ model.

Specifically, oscillations in the presence of a single sterile neutrino can be modelled by extending the standard picture to include four neutrino eigenstates. In this case, six new parameters are introduced in the model: one additional mass square difference $\Delta m_{41}^{2}$, three active-sterile mixing angles $\theta_{14}, \theta_{24}$ and $\theta_{34}$, and two additional CP-violating phases $\delta_{14}, \delta_{24}$.

The neutrino evolution in matter can be described by the following effective Hamiltonian:

$$
H=U H_{0} U^{\dagger}+V
$$

where $H_{0}=\operatorname{diag}\left(0, \Delta m_{21}^{2}, \Delta m_{31}^{2}, \Delta m_{41}^{2}\right) / 2 E$, and $V=\sqrt{2} G_{F} \operatorname{diag}\left(N_{e}, 0,0, N_{n} / 2\right)$, with $G_{F}$ being the Fermi constant and $N_{e}, N_{n}$ representing the density of electrons and neutrons in the propagation medium. $U$ is an extended $4 \times 4$ unitary matrix relating flavour and mass eigenstates, which can be parametrised such that:

$$
U=R_{34} \tilde{R}_{24} \tilde{R}_{14} R_{23} \tilde{R}_{13} R_{12},
$$

where $R_{j k}$ is a rotation matrix in the $j-k$ plane and, similarly, $\tilde{R}_{j k}$ is a generalised unitary rotation matrix with an added complex phase.

In the $3+1$ model, the active-sterile mixing elements are expressed by

$$
\begin{aligned}
U_{e 4} & =\sin \theta_{14} e^{-i \delta_{14}}, \\
U_{\mu 4} & =\cos \theta_{14} \sin \theta_{24} e^{-i \delta_{24}}, \\
U_{\tau 4} & =\cos \theta_{14} \cos \theta_{24} \sin \theta_{34} .
\end{aligned}
$$

Several experiments have been searching for the SBL sterile neutrino. To date, results are not fully consistent with the $3+1$ model: disappearance experiments results are compatible with the standard neutrino scenario while some appearance experiments, such as LSND [11] and MiniBooNE [12], observed significant $\nu_{e}$ or $\bar{\nu}_{e}$ excesses. The global fit of the experimental data with the $3+1$ model results in a poor goodness-of-fit, suggesting the need of additional factors in order to explain all data.

Even stronger bounds on the sterile parametric space come from cosmology [13], which indirectly constrains the effective number of relativistic species $N_{\text {eff }}$ in our Universe. Theoretically, the three active neutrinos give $N_{\text {eff }} \sim 3$ [14]. If a light sterile neutrino with the mixing parameters determined by SBL oscillations is included in the model, it should have been fully thermalised with the active neutrinos [15]. This would require $N_{\text {eff }} \sim 4$. Cosmological data measure a value of $N_{\text {eff }}$ well-compatible with three neutrino species [16], showing a tension with the SBL anomalies. Such a tension is relaxed when cosmological data are combined with astrophysical measurements of cepheids, supernovae and gravitational lensing. In this case, the obtained value of $N_{\text {eff }}$ is compatible with four at $68 \%$ C.L. $[15,16]$.

More generally, cosmological data alone can be compatible with a sterile neutrino with a mass in the $\mathrm{eV}$ range only if its contribution to $N_{\text {eff }}$ is very small, or with a somewhat larger $N_{\text {eff }}$ only if it comes from a nearly massless sterile particle $[17,18]$. 
Therefore, more terrestrial and cosmological observations are necessary to understand the origin of the SBL anomalies. Moreover, new observations able to constrain the notfully-excluded sterile neutrino region from cosmology, at very low sterile mass splittings $\left(\Delta m_{41}^{2} \ll 1 \mathrm{eV}^{2}\right)$ can further contribute to testing the sterile neutrino hypothesis.

In this context, the role of next-generation neutrino detectors, such as KM3NeT, is relevant, given their ability to probe the sterile neutrino hypothesis with atmospheric neutrinos [19, 20]. KM3NeT is a research infrastructure hosting a network of next generation neutrino telescopes currently under construction in the Mediterranean Sea [2] and built upon the experience from the ANTARES neutrino telescope [21]. Once completed, KM3NeT will consist of two detectors: (1) ORCA (Oscillation Research with Cosmics in the Abyss) near Toulon, France, optimised for GeV-scale atmospheric neutrino studies, and (2) ARCA (Astroparticle Research with Cosmics in the Abyss), in Sicily, Italy, optimised for the observation of higher-energy $\left(E_{\nu}>1 \mathrm{TeV}\right)$ neutrinos from astrophysical sources.

By exploiting the natural source of atmospheric neutrinos, passing through the Earth and interacting within the detector volume, KM3NeT will perform neutrino oscillation studies over a broad range of energies (from few $\mathrm{GeV}$ up to $\mathrm{PeV}$ ) and baselines (up to the Earth diameter). Matter effects, experienced by atmospheric neutrinos during their passage through the Earth, are expected to enhance the effect of the presence of a sterile neutrino. Moreover, the wide L/E range available in KM3NeT increases its potential to investigate the existence of a sterile neutrino in the $3+1$ model.

This paper is focused on the ORCA capability to search for a light sterile neutrino. It will be shown that ORCA has a high potential to simultaneously constrain the activesterile mixing angles $\theta_{14}, \theta_{24}, \theta_{34}$ and the effective angle $\theta_{\mu e}$, with three years of data taking. Particularly, the ORCA sensitivity to such parameters is competitive with other experiments for sterile neutrino mass at the $\mathrm{eV}$ scale, indicated by SBL anomalies, and it is able to provide even stronger constraints for extremely low sterile mass splittings $\left(\Delta m_{41}^{2}\right.$ down to $10^{-5} \mathrm{eV}^{2}$ ).

This paper is organised as follows: section 2 describes the KM3NeT/ORCA neutrino telescope. Section 3 discusses the $3+1$ flavour model and oscillation probabilities. Section 4 describes the sterile neutrino analysis method, including a brief summary of the ORCA Monte Carlo (MC) simulation flow. Results on the ORCA sensitivity are presented in section 5. Finally, the results are summarised and discussed in section 6 .

\section{The KM3NeT/ORCA detector}

KM3NeT/ORCA is a deep water neutrino detector under construction in the Mediterranean Sea. Its location is $42^{\circ} 48^{\prime} \mathrm{N} 06^{\circ} 02^{\prime} \mathrm{E}$, about $40 \mathrm{~km}$ offshore from Toulon, France, at a depth of about $2450 \mathrm{~m}$. Upon its completion, ORCA will consist of 115 flexible detection units (DUs), $200 \mathrm{~m}$ high, each comprising 18 Digital Optical Modules (DOMs). A DOM is a pressure resistant, 17-inch diameter glass sphere containing a total of $31,3^{\mathrm{n}}$ photomultiplier tubes (PMTs) and their associated electronics.

The primary goal of ORCA is to determine the neutrino mass ordering and to make neutrino oscillation measurements, such as atmospheric parameters $\left(\sin ^{2} \theta_{23}, \Delta m_{31}^{2}\right)$ as 
well as to search for $\nu_{\tau}$ appearance [22]. Neutrino oscillation studies [2] have demonstrated the presence of a resonance in neutrino oscillation probabilities for few-GeV $(2-8 \mathrm{GeV})$ atmospheric neutrinos passing through the Earth. Such a resonance allows the NMO [2] measurement.

The ORCA geometrical configuration is optimised for studies with atmospheric neutrinos in the few $\mathrm{GeV}$ range: the horizontal spacing between DUs is $\sim 20 \mathrm{~m}$, whereas the vertical spacing between DOMs in each DU is $\sim 9 \mathrm{~m}$, with the first DOM being about $30 \mathrm{~m}$ above the seabed. The total instrumented volume is $6.7 \cdot 10^{6} \mathrm{~m}^{3}$ (about $7 \mathrm{Mt}$ of sea water).

In this energy regime, the events produced by atmospheric neutrinos interacting in water are spatially contained. In particular, two event topologies can be produced: track-like events, characterised by a long muon track, mostly from $\nu_{\mu}$ charged-current (CC) interactions in water, and shower-like events, characterised by events with no distinguishable tracks, mostly from $\nu_{e}-\mathrm{CC}$ and all neutral-current $(\mathrm{NC})$ interactions, but with sizeable contributions from $\nu_{\tau^{-}} \mathrm{CC}$ and $\nu_{\mu}$-CC events with short tracks. A track-like event in water has a length of $\sim 4 \mathrm{~m} / \mathrm{GeV}$, whereas shower-like events have a $\log (\mathrm{E} / \mathrm{GeV})$ dependence, which corresponds to a size of the order of a few meters.

The ORCA detector is an excellent instrument for the sterile neutrino search due to its dense configuration and to matter effects, whose impact in oscillation probabilities of $\mathrm{GeV}$ neutrinos travelling in the Earth is described in the next section.

More details on KM3NeT/ORCA can be found in [2, 22].

\section{Theoretical background}

The general solutions to the Hamiltonian in eq. (1.1) have a rich phenomenology that is difficult to express in analytical form. For the purposes of this analysis, eq. (1.1) is solved numerically in its full form using the software package OscProb [23]. Figure 1 shows an example of the impact of the matter potential on the effective values of the squared masses (eigenvalues of eq. (1.1)) as a function of energy, assuming a medium of constant density for illustration purposes. Four resonances can be identified in the sterile neutrino models as regions of minimal distance between consecutive masses: one related to each pair $\left(s_{1 k},{ }^{1}\right.$ $\Delta m_{k 1}^{2}$ ) (at $\sim 0.05 \mathrm{GeV}, 4 \mathrm{GeV}$ and $3 \mathrm{TeV}$ ), and a second-order resonance connecting $s_{23}$, $s_{24}, s_{34}$, and $\Delta m_{31}^{2}$ (at $\sim 100 \mathrm{GeV}$ ), and with a strong dependence on $\delta_{24}$ as explained in section 3.1.2. The eigenvectors of eq. (1.1) define an effective mixing matrix which, in a medium of constant density, can be used to compute oscillation probabilities by direct replacement in the vacuum oscillation formula:

$$
\begin{aligned}
P_{\alpha \beta}= & \delta_{\alpha \beta}-\sum_{j>k} 4 \operatorname{Re}\left[U_{\alpha j} U_{\beta j}^{*} U_{\alpha k}^{*} U_{\beta k}\right] \sin ^{2} \frac{\Delta_{j k} L}{2} \\
& -\sum_{j>k} 2 \operatorname{Im}\left[U_{\alpha j} U_{\beta j}^{*} U_{\alpha k}^{*} U_{\beta k}\right] \sin \Delta_{j k} L
\end{aligned}
$$

where $\Delta_{j k}=\Delta m_{j k}^{2} / 2 E$. Figure 2 shows examples of effective values of the magnitude of some terms from eq. (3.1) as a function of neutrino energy. The impact of the aforemen-

\footnotetext{
${ }^{1} s_{j k}, c_{j k}$ represent $\sin \theta_{j k}$ and $\cos \theta_{j k}$ respectively.
} 

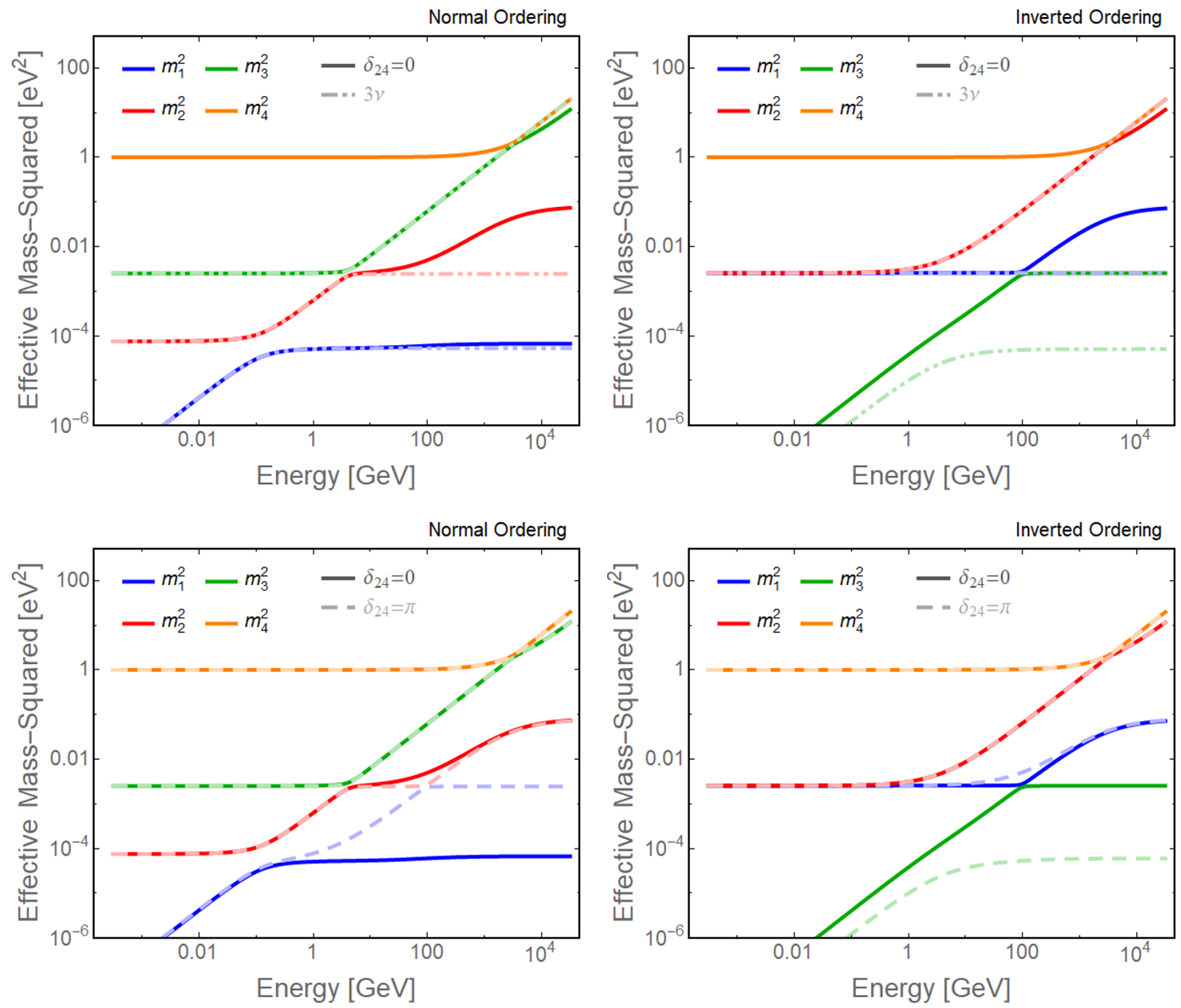

Figure 1. Effective mass-squared values, representing the eigenvalues of eq. (1.1) for neutrinos in both normal (left) and inverted (right) orderings, as a function of neutrino energy. Three models are shown: in the upper panels, the standard picture with three active neutrinos $(3 \nu)$, is compared to a model with one light sterile neutrino where the CP violating phase $\delta_{24}$ is set to 0 . In the lower panels, two sterile neutrino models are compared with $\delta_{24}$ set to either 0 or $\pi$. The absolute mass scale has been chosen so that the lightest neutrino is massless in vacuum. The oscillation parameters were set to $\Delta m_{21}^{2}=7.5 \times 10^{-5} \mathrm{eV}^{2},\left|\Delta m_{31}^{2}\right|=2.5 \times 10^{-3} \mathrm{eV}^{2},\left|\Delta m_{41}^{2}\right|=1 \mathrm{eV}^{2}, s_{12}^{2}=0.3, s_{13}^{2}=0.02$, $s_{23}^{2}=0.57, s_{14}^{2}=0.01, s_{24}^{2}=s_{34}^{2}=0.04$, and $\delta_{13}=\delta_{14}=0$. The matter density is set to $8.5 \mathrm{~g} / \mathrm{cm}^{3}$ with a ratio $N_{n} / N_{e}=1.08$.

tioned resonances can be readily identified. While the full numerical solutions exemplified above can already provide some insight, some exploration of common analytical approximations can be enlightening even if not used in the analysis. They are described in the following subsections. 

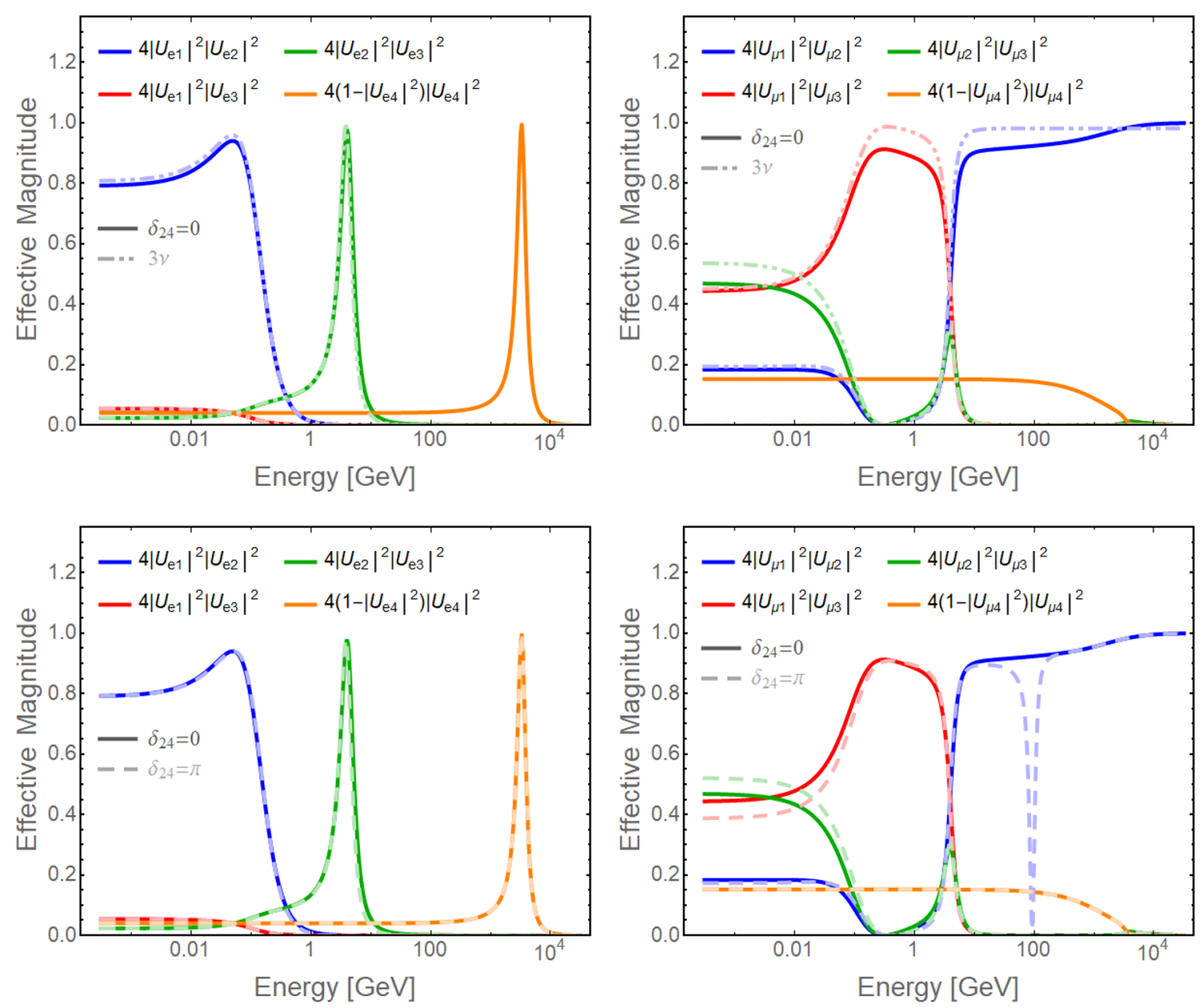

Figure 2. Effective oscillation magnitudes associated with $\Delta_{21}, \Delta_{31}, \Delta_{32}$, and $\Delta_{4 k}$ (see equation (3.1)) as a function of neutrino energy. The latter is taken as a combination of all three mass-squared difference terms involving the fourth mass state, which are approximately of equal frequency at this scale. Left: magnitudes associated with $\nu_{e}$ disappearance probabilities. Right: magnitudes associated with $\nu_{\mu}$ disappearance probabilities. Top: comparison between $3 \nu$ and a sterile neutrino scenario with $\delta_{24}=0$. Bottom: comparison between sterile neutrino scenarios with $\delta_{24}$ set to either 0 or $\pi$. All plots apply to neutrinos in normal ordering. The same parameters as in figure 1 were used.

\subsection{Large $\left|\Delta m_{41}^{2}\right|$ limit}

Anomalous oscillation results, such as LSND and MiniBooNE, are commonly interpreted as oscillations in a higher frequency than the solar and atmospheric scales. Under this scenario, the limit $\Delta m_{41}^{2} \rightarrow \infty$ can be considered in which all oscillations driven by $\Delta m_{41}^{2}$ are averaged out and observable only through scaling factors. Hereafter, this will be referred to as the high frequency (HF) region.

Following ref. [24], the mixing matrix $U$ can be split such that $U=U^{4 \nu} U^{3 \nu}$, with $U^{3 \nu}=R_{23} \tilde{R}_{13} R_{12}$ containing only the active-active mixing elements, and $U^{4 \nu}=R_{34} \tilde{R}_{24} \tilde{R}_{14}$ 
representing the active-sterile mixing. If the Hamiltonian is rotated with $U^{4 \nu}$, it becomes approximately block-diagonal in the limit where $\Delta m_{41}^{2} \rightarrow \infty$ :

$$
\tilde{H}=U^{3 \nu} H_{0}\left(U^{3 \nu}\right)^{\dagger}+\left(U^{4 \nu}\right)^{\dagger} V U^{4 \nu} \approx\left(\begin{array}{cc}
\tilde{H}^{(3)} & 0 \\
0 & \Delta_{41}
\end{array}\right)
$$

The evolution matrix can then be expressed as:

$$
S \approx U^{4 \nu}\left(\begin{array}{cc}
e^{-i \tilde{H}^{(3)} L} & 0 \\
0 & e^{-i \Delta_{41} L}
\end{array}\right)\left(U^{4 \nu}\right)^{\dagger}
$$

The remaining problem lies in the diagonalisation of $\tilde{H}^{(3)}$. For that, further approximations, which are valid in specific energy regimes, are employed. In general, a scale $\epsilon$ will be used to represent small quantities. The mixing parameters $s_{34}, s_{24}, s_{14}$, and $s_{13}$ will all be considered of $\mathcal{O}(\epsilon)$. Additionally, $\Delta m_{21}^{2} / \Delta m_{31}^{2} \sim s_{13}^{2}$ will be treated as $\mathcal{O}\left(\epsilon^{2}\right)$. In this approximation, probabilities can be written to $\mathcal{O}\left(\epsilon^{2}\right)$ as:

$$
\begin{aligned}
P_{e e} & \approx P_{e e}^{(3)} \cos 2 \theta_{14}, \\
P_{e \mu} & \approx c_{14}^{2} c_{24}^{2} P_{e \mu}^{(3)}+2 c_{14}^{2} \operatorname{Re}\left[U_{\mu 2}^{4 \nu} U_{\mu 1}^{4 \nu^{*}} S_{e \mu}^{(3)} S_{e e}^{(3)^{*}}\right], \\
P_{\mu e} & \approx c_{14}^{2} c_{24}^{2} P_{\mu e}^{(3)}+2 c_{14}^{2} \operatorname{Re}\left[U_{\mu 2}^{4 \nu} U_{\mu 1}^{4 \nu^{*}} S_{\mu e}^{(3)} S_{e e}^{(3)^{*}}\right] \\
P_{\mu \mu} & \approx P_{\mu \mu}^{(3)} \cos 2 \theta_{24}+2 c_{24}^{2} \operatorname{Re}\left[U_{\mu 2}^{4 \nu} U_{\mu 1}^{4 \nu *} S_{\mu \mu}^{(3)}\left(S_{e \mu}^{(3)^{*}}+S_{\mu e}^{(3)^{*}}\right)\right]
\end{aligned}
$$

where $U_{\mu 1}^{4 \nu}=-s_{14} s_{24} e^{i \delta_{14}-i \delta_{24}}, U_{\mu 2}^{4 \nu}=c_{24}$, and $S_{\alpha \beta}$ correspond to elements of the evolution matrix in eq. (3.3). The effect of mixing with sterile neutrinos is given by a scaling of the 3-neutrino submatrix probabilities. Additionally, some interference terms appear if both $s_{14}$ and $s_{24}$ are non-zero.

\subsubsection{The ORCA low energy regime}

The ORCA detector is most sensitive to neutrinos in the energy range of $3-100 \mathrm{GeV}$ considered in this analysis, crossing the Earth with paths of mean density varying between 3 and $9 \mathrm{~g} / \mathrm{cm}^{3}$. In the lower part of this energy range $(E<10 \mathrm{GeV})$, when $V_{e}=\sqrt{2} G_{F} N_{e} \sim$ $\Delta_{31}=\Delta m_{31}^{2} / 2 E$, to leading order in small quantities, $\tilde{H}^{(3)}$ simplifies to:

$$
\tilde{H}^{(3)} \approx R_{23}\left(\begin{array}{ccc}
V_{e} & 0 & \Delta_{31} c_{13} s_{13} e^{-i \delta_{13}} \\
0 & 0 & 0 \\
\Delta_{31} c_{13} s_{13} e^{i \delta_{13}} & 0 & \Delta_{31} c_{13}^{2}
\end{array}\right) R_{23}^{\dagger} .
$$


This approximately 2-flavour form can be readily solved leading to the well-known MSW resonance of $\theta_{13}$ :

$$
\begin{aligned}
\tilde{H}^{(3)} & \approx R_{23} \tilde{R}_{13}^{m} \cdot\left(\begin{array}{ccc}
-\Delta_{31}^{m} / 2 & 0 & 0 \\
0 & 0 & 0 \\
0 & 0 & \Delta_{31}^{m} / 2
\end{array}\right)\left(\tilde{R}_{13}^{m}\right)^{\dagger} R_{23}^{\dagger}+\text { const. } \\
\Delta_{31}^{m} & =\sqrt{\left(\Delta_{31} \cos 2 \theta_{13}-V_{e}\right)^{2}+\Delta_{31}^{2} \sin ^{2} 2 \theta_{13}}, \\
\sin 2 \theta_{13}^{m} & =\frac{\left|\Delta_{31}\right|}{\Delta_{13}^{m}} \sin 2 \theta_{13},
\end{aligned}
$$

where $\tilde{R}_{13}^{m}$ represents the effective generalised unitary rotation matrix in the 1-3 plane, parametrised by the effective mixing angle $\theta_{13}^{m}$ and the unchanged phase $\delta_{13}$.

All effects arising from the presence of sterile neutrino are constrained to vacuum-like mixing through $U^{4 \nu}$ as in eq. (3.3).

\subsubsection{The ORCA high energy regime}

At higher energies $(E \gtrsim 10 \mathrm{GeV})$, the matter potential starts to dominate. However, a new resonance can still be found when $\Delta_{31} / V_{n}$ is of $\mathcal{O}\left(\epsilon^{2}\right)$. In this regime, $\tilde{H}^{(3)}$ is expressed in leading order as:

$$
\tilde{H}^{(3)} \approx\left(\begin{array}{ccc}
V_{e} & 0 & 0 \\
0 & \Delta_{31} s_{23}^{2}+V_{n}\left|U_{s 2}^{4 \nu}\right|^{2} & \Delta_{31} s_{23} c_{23}+V_{n} U_{s 2}^{4 \nu^{*}} U_{s 3}^{4 \nu} \\
0 & \Delta_{31} s_{23} c_{23}+V_{n} U_{s 2}^{4 \nu} U_{s 3}^{4 \nu} & \Delta_{31} c_{23}^{2}+V_{n}\left|U_{s 3}^{4 \nu}\right|^{2}
\end{array}\right),
$$

where $U_{s 2}^{4 \nu}=-c_{34} s_{24} e^{i \delta_{24}}$ and $U_{s 3}^{4 \nu}=-s_{34}$. Once again, the Hamiltonian is approximately block diagonal and can be easily solved to give:

$$
\begin{aligned}
\tilde{H}^{(3)} \approx & R_{23}^{m}\left(\begin{array}{ccc}
V_{e} & 0 & 0 \\
0 & -\Delta_{32}^{m} / 2 & 0 \\
0 & 0 & \Delta_{32}^{m} / 2
\end{array}\right)\left(R_{23}^{m}\right)^{\dagger}+\text { const., } \\
\Delta_{32}^{m 2}= & {\left[\Delta_{31} \cos 2 \theta_{23}+\left(\left|U_{s 3}^{4 \nu}\right|^{2}-\left|U_{s 2}^{4 \nu}\right|^{2}\right) V_{n}\right]^{2} } \\
& +\left|\Delta_{31} \sin 2 \theta_{23}+2 V_{n} U_{s 2}^{4 \nu^{*}} U_{s 3}^{4 \nu}\right|^{2}, \\
\sin 2 \theta_{23}^{m}= & \frac{1}{\Delta_{32}^{m}}\left|\Delta_{31} \sin 2 \theta_{23}+2 V_{n} U_{s 2}^{4 \nu *} U_{s 3}^{4 \nu}\right| .
\end{aligned}
$$

This new resonance corresponds to a second order effect that couples the $2-3$ sector indirectly via $s_{24}$ and $s_{34}$. It provides a very rich structure having two main features: a resonance when $\sin 2 \theta_{23}^{m} \rightarrow 1$ and an antiresonance when $\sin 2 \theta_{23}^{m} \rightarrow 0$ at finite $V_{n}$. The resonance conditions are:

$$
\begin{aligned}
& V_{n}=\frac{\Delta_{31} \cos 2 \theta_{23}}{\left(\left|U_{s 2}^{4 \nu}\right|^{2}-\left|U_{s 3}^{4 \nu}\right|^{2}\right)} \Rightarrow \sin 2 \theta_{23}^{m}=1, \\
& V_{n}=-\frac{\Delta_{31} \sin 2 \theta_{23}}{2 U_{s 2}^{4 \nu^{*}} U_{s 3}^{4 \nu}} \Rightarrow \sin 2 \theta_{23}^{m}=0 .
\end{aligned}
$$



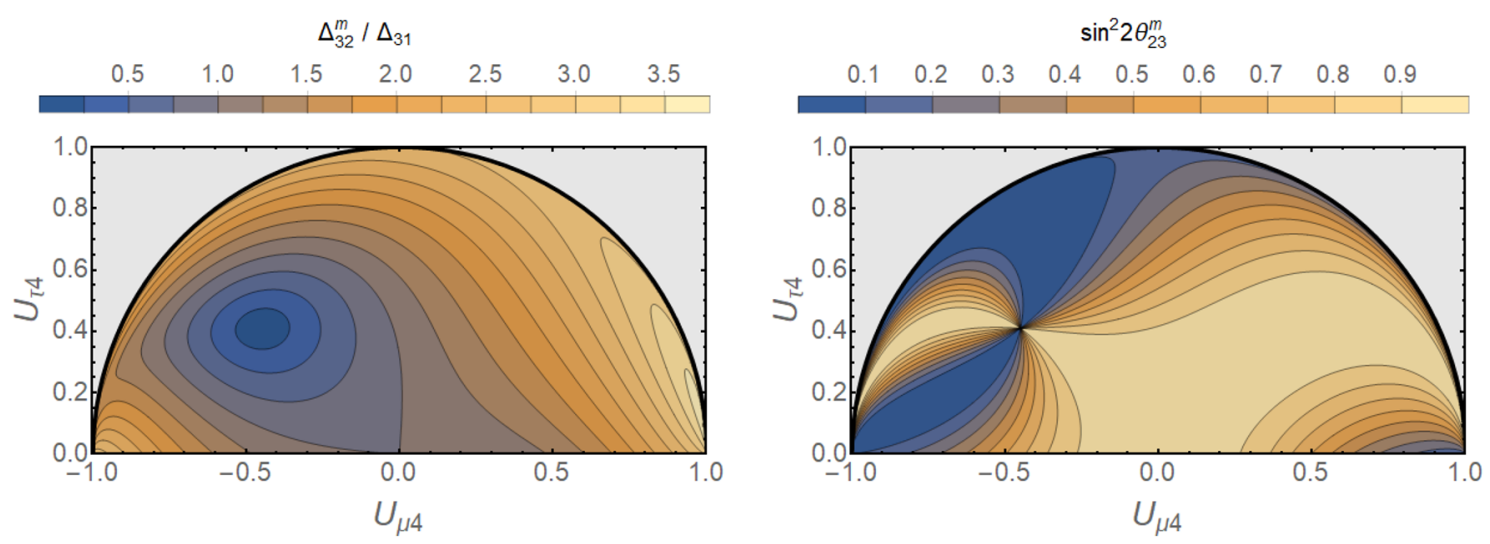

Figure 3. Effective parameters $\Delta_{32}^{m} / \Delta_{31}$ (left) and $\sin ^{2} 2 \theta_{23}^{m}$ (right) as a function of real values of $U_{\mu 4}$ and $U_{\tau 4}$, for a neutrino energy of $20 \mathrm{GeV}$. Here, $\Delta m_{31}^{2}=2.5 \times 10^{-3} \mathrm{eV}^{2}, s_{23}^{2}=0.57$, and a matter density of $8.5 \mathrm{~g} / \mathrm{cm}^{3}$ with a ratio $N_{n} / N_{e}=1.08$ were assumed. The resonance and antiresonance described in equations (3.16) and (3.17) are visible on the right as regions of maximum and minimum $\sin ^{2} 2 \theta_{23}^{m}$. At the point where they seem to meet, a pole exists where $\Delta_{32}^{m} \rightarrow 0$ and $\sin ^{2} 2 \theta_{23}^{m}$ becomes undefined.

A pole exists when both conditions are satisfied, as $\Delta_{32}^{m} \rightarrow 0$ and no mixing is possible. The structure of these resonances is shown in figure 3. Since $\theta_{23}$ is close to maximal, the antiresonance of eq. (3.17) is the most noticeable effect in this regime. The antiresonance occurs for neutrinos when $\cos \delta_{24} \Delta m_{31}^{2}<0$ or for antineutrinos when $\cos \delta_{24} \Delta m_{31}^{2}>0$, and is only exact for $\delta_{24}=0$ or $\pi$. Hence, there is a degeneracy between mass ordering and $\operatorname{sign}\left(\cos \delta_{24}\right)$, enhanced by the maximal value of $\sin 2 \theta_{23}$, which suppresses NMO contributions from the resonance term in eq. (3.16).

\subsection{Finite $\left|\Delta m_{41}^{2}\right|$ regime}

At values of $\Delta m_{41}^{2}$ for which the associated oscillations cannot be averaged out, no simplifying approximations are known to us at the time of writing. In ORCA, this corresponds to values of $\Delta m_{41}^{2} \lesssim 0.1 \mathrm{eV}^{2}$, this regime will be referred to as the low frequency (LF) region. In this case, many interference terms are present and the probability formulas can become exceedingly complex. Nevertheless, a full numerical solution is possible on all regimes considered in the analysis, and it is used to extend the results through six orders of magnitude in $\Delta m_{41}^{2}$. For simplicity, $\Delta m_{41}^{2}$ will be restricted to positive values.

\section{Sterile neutrino analysis}

The analysis presented here is based on detailed Monte Carlo (MC) simulations as described in ref. [22]. Neutrino interactions are generated with gSeaGen [25], which is based on GENIE [26]. Secondary particles and their emitted Cherenkov light are propagated with KM3Sim [27], a software package based on GEANT4 [28]. The atmospheric neutrino flux is computed from the Honda model [29] for the Gran Sasso site without mountain over 


\begin{tabular}{|c|c|c|c|c|}
\hline & $E[\mathrm{GeV}]$ & $\cos \theta_{Z}$ & $E^{\prime}[\mathrm{GeV}]$ & $\cos \theta_{Z}^{\prime}$ \\
\hline Bins & 40 & 40 & 20 & 20 \\
\hline Range & {$[1,100]$} & {$[-1,0]$} & {$[2,100]$} & {$[-1,0]$} \\
\hline
\end{tabular}

Table 1. Bin choice for the MC-based response matrix, $R$, used in this analysis. Energy bins are in $\log _{10}$ space.

the detector, assuming minimum solar activity. Atmospheric muons are generated with MUPAGE [30, 31], and propagated with KM3 [32].

Event reconstruction is performed via a maximum likelihood fit to shower and track hypotheses. Background events arising from noise and atmospheric muons are rejected with two independent Random Decision Forests (RDF) trained on MC simulations. A third RDF was used to separate neutrino candidates into three topology classes defined by the output score of the RDF, trained to identify track-like events. Events with a track score larger than 0.7 are labelled as track-like, track scores less than 0.3 are labelled as shower-like, and other values are labelled as an intermediate topology. Moreover, as in ref. [22], only upgoing events are considered in order to get rid of the atmospheric muon contamination.

Instead of using parametrised response functions as in ref. [22], the analysis reported here is based on the aforementioned MC simulations to directly model the detector response. The two approaches have been compared and found consistent.

The MC-based modelling of the detector response is implemented in the KM3NeT framework Swim [33]. The detector response is represented by a 4-dimensional matrix, as a function of true and reconstructed neutrino energy $E, E^{\prime}$, and zenith angle $\theta, \theta^{\prime}$, for each interaction channel $\nu_{x}, R^{\left[\nu_{x} \rightarrow i\right]}\left(E, \theta, E^{\prime}, \theta^{\prime}\right)$. Each entry of this matrix summarises in a single dimensionless coefficient the efficiency of detection, classification and probability of reconstruction for a given true bin $(E, \theta)$. Therefore, $R$ incorporates all the effects related both to the detector and to the event selection. More details on this approach, can be found in ref. [33]. The binning scheme, for the detector response matrix, used in this analysis is shown in table 1. Since the atmospheric neutrino flux follows a power law in energy, equalwidth bins in $\log _{10} E$ are chosen. The same choice is adopted for reconstructed events histograms, as the relative energy resolution in ORCA is, to first order, constant above $\sim 10 \mathrm{GeV}, \delta E / E \simeq \delta\left(\log _{10} E\right) \simeq 15 \%[34]$.

A binning of constant width in $\cos \theta_{Z}$ is used. This is motivated by the fact that the solid angle covered by an interval of zenith angle $\theta_{1} \leq \theta \leq \theta_{2}$ is proportional to $\left|\cos \theta_{1}-\cos \theta_{2}\right|$ and, considering to first order the atmospheric neutrino flux as isotropic, this choice yields equally populated bins along the zenith angle axis.

For reconstructed event histograms, the choice of binning granularity is dominated by the detector resolutions. The bin width should be comparable with the typical error on the reconstructed variable. Moreover, it should account for a sufficiently smooth sampling of the detector response, to minimise the finite MC statistics issues, which can result in overestimations of sensitivity [33]. Statistical fluctuations due to the sparse MC effect are taken into account by following the "Beeston and Barlow method" [35]. 


\begin{tabular}{|c|c|c|c|c|c|c|}
\hline & $\sin ^{2} \theta_{12}$ & $\sin ^{2} \theta_{23}$ & $\sin ^{2} \theta_{13}$ & $\delta_{\mathrm{CP}}$ & $\Delta m_{21}^{2}\left(\mathrm{eV}^{2}\right)$ & $\Delta m_{31}^{2}\left(\mathrm{eV}^{2}\right)$ \\
\hline NO & 0.310 & 0.563 & 0.02237 & $221^{\circ}$ & $7.39 \times 10^{-5}$ & $2.528 \times 10^{-3}$ \\
\hline IO & 0.310 & 0.565 & 0.02259 & $282^{\circ}$ & $7.39 \times 10^{-5}$ & $-2.510 \times 10^{-3}$ \\
\hline
\end{tabular}

Table 2. Benchmark oscillation parameters for NO and IO, taken from the NuFit v4.1 result [36].
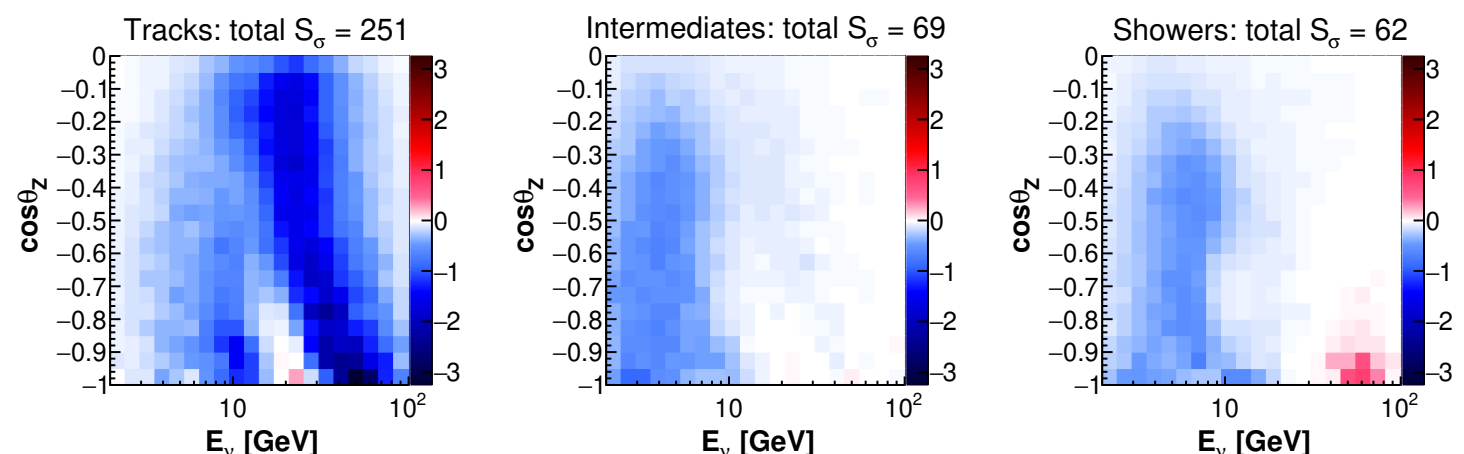

Figure 4. $S_{\sigma}$ distribution of the three topologies considered in the analysis (tracks, intermediates and showers) assuming three years of data taking. The colour scale denotes the $S_{\sigma}$ value for each bin, whereas the total $S_{\sigma}$ is reported on top of the plots: the high value obtained is due to the normalisation. The sterile neutrino parameters are $\sin ^{2} \theta_{14}=0, \sin ^{2} \theta_{24}=0.03, \sin ^{2} \theta_{34}=0.05$, $\Delta m_{41}^{2}=1 \mathrm{eV}^{2}$.

The values of the standard neutrino parameters used in this analysis is taken from the NuFit v4.1 global fit result with Super-Kamiokande (SK) data [36] and summarised in table 2, for both normal (NO) and inverted ordering (IO). Current fits have large errors on $\delta_{C P}$. The impact of such variable in the analysis has been tested and found to be negligible. For this reason, its value is fixed to the ones reported in table 2. Moreover, $\Delta m_{41}^{2}>0$ is always assumed. Oscillation probabilities are evaluated with the software package OscProb [23], and to account for Earth's matter effects the PREM model [37] with 44 layers is used.

The above information can be used to define the distinguishability $S_{\sigma}$, as a quick estimator of sensitivity of measurements, with the goal of illustrating the impact of a sterile neutrino in the event distributions, as

$$
S_{\sigma}=\frac{\left(N_{\text {Sterile }}-N_{\text {Standard }}\right)\left|N_{\text {Sterile }}-N_{\text {Standard }}\right|}{N_{\text {Sterile }}},
$$

where $N_{\text {Sterile }}$ and $N_{\text {Standard }}$ are the number of events, as a function of reconstructed energy and zenith angle, in the sterile and standard hypothesis respectively.

Figure 4 shows the distinguishability distribution for a sterile neutrino in the HF region, for non-zero $\theta_{24}$ and $\theta_{34}$, assuming three years of ORCA data taking. The presence of the sterile neutrino mainly impacts the track-like events in the form of a deficit of upgoing events at higher energies $\left(E^{\prime} \geq 40 \mathrm{GeV}\right)$. Therefore, this region of the sterile parameter space can be well constrained also by neutrino telescopes whose energy threshold is higher than that of ORCA, such as ANTARES [38] and IceCube/DeepCore [39]. 

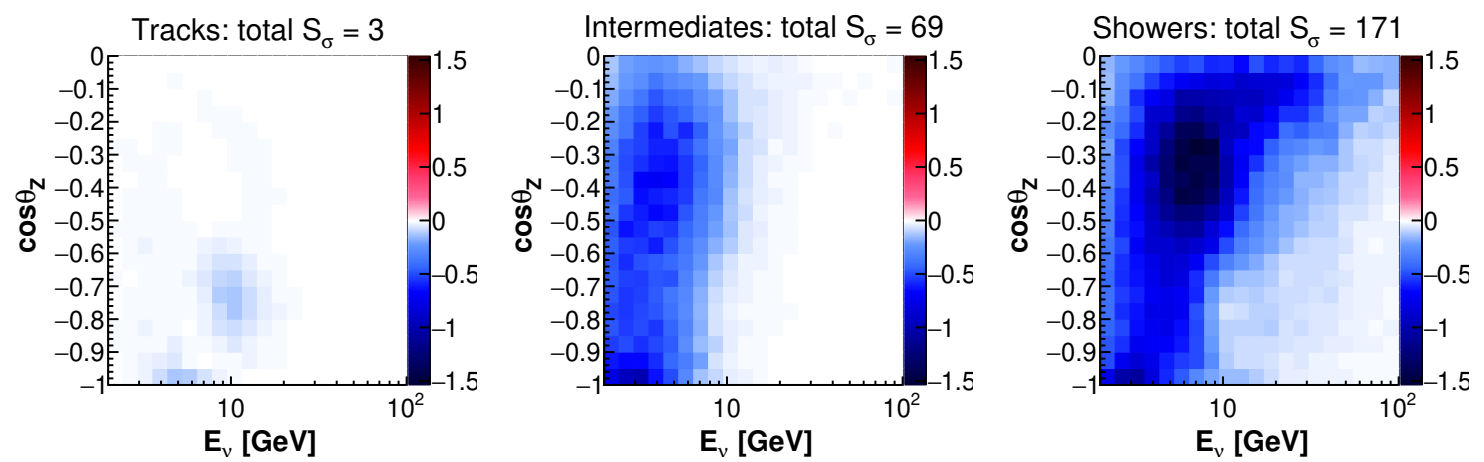

Figure 5. $S_{\sigma}$ distribution of the three topologies considered in the analysis (tracks, intermediates and showers) assuming three years of data taking. The colour scale denotes the $S_{\sigma}$ value for each bin, whereas the total $S_{\sigma}$ is reported on top of the plots: the high value obtained is due to the normalisation. The sterile neutrino parameters are $\sin ^{2} \theta_{14}=0.05, \sin ^{2} \theta_{24}=\sin ^{2} \theta_{34}=0$, $\Delta m_{41}^{2}=1 \mathrm{eV}^{2}$.
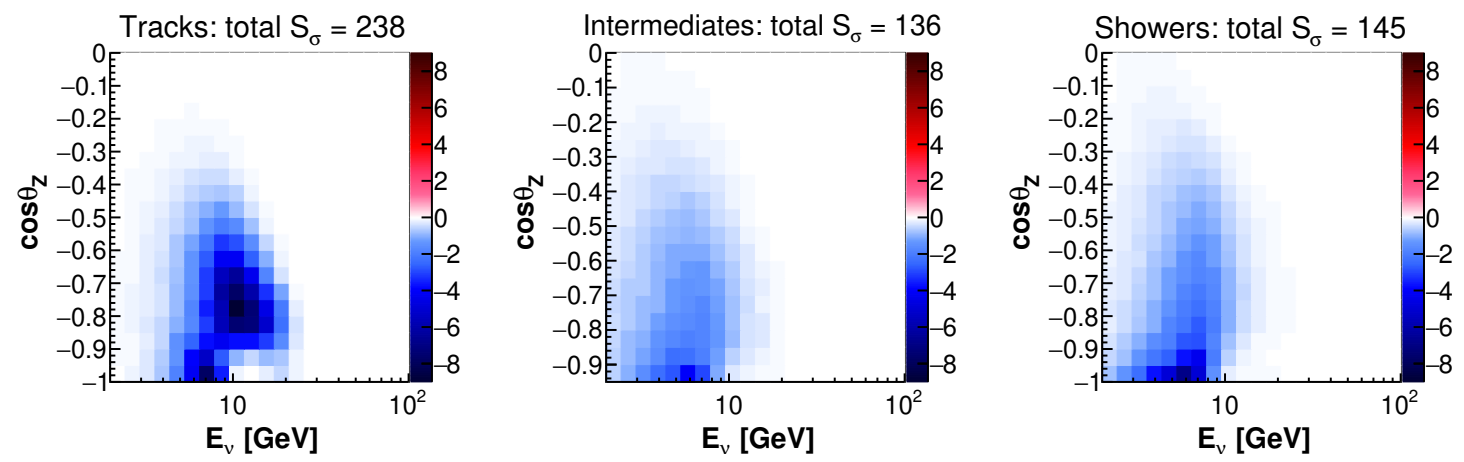

Figure 6. $S_{\sigma}$ distribution of the three topologies considered in the analysis (tracks, intermediates and showers) assuming three years of data taking. The colour scale denotes the $S_{\sigma}$ value for each bin, whereas the total $S_{\sigma}$ is reported on top of the plots: the high value obtained is due to the normalisation. The sterile neutrino parameters are $\sin ^{2} \theta_{14}=\sin ^{2} \theta_{24}=\sin ^{2} \theta_{34}=0.01$, $\Delta m_{41}^{2}=10^{-4} \mathrm{eV}^{2}$.

To understand the sensitivity to $\theta_{14}$ in particular, the distinguishability for a sterile neutrino in the HF region and $\sin ^{2} \theta_{14}=0.05$ is shown in figure 5 . In this case, the showerlike events are the most affected and mainly for energies $<20 \mathrm{GeV}$. It follows that ORCA is well suited to test $\theta_{14}$.

Finally, figure 6 shows the impact of a sterile neutrino with $\Delta m_{41}^{2}=10^{-4} \mathrm{eV}^{2}$. In this case, the energy region $E^{\prime}<10 \mathrm{GeV}$ is the most significant, and all the three event topologies are highly impacted. This applies also for $\Delta m_{41}^{2}=10^{-2}, 10^{-3}, 10^{-5} \mathrm{eV}^{2}$.

The sensitivity evaluation is based on the minimisation of a negative log-likelihood function describing the agreement between a model prediction and observed data. This is done with the Asimov approach [40] assuming the negative log-likelihood follows a chi- 


\begin{tabular}{|c|c|}
\hline Parameter & Gaussian Prior $(\mu \pm \sigma)$ \\
\hline$\nu_{e} / \bar{\nu}_{e}$ & $0 \pm 0.07$ \\
\hline$\nu_{\mu} / \bar{\nu}_{\mu}$ & $0 \pm 0.05$ \\
\hline$\nu_{e} / \nu_{\mu}$ & $0 \pm 0.02$ \\
\hline NC Scale & No prior \\
\hline Energy Scale & $1 \pm 0.05$ \\
\hline Energy Slope & No prior \\
\hline Zenith Angle Slope & $0 \pm 0.02$ \\
\hline Track Normalisation & No Prior \\
\hline Intermediate Normalisation & No Prior \\
\hline Shower Normalisation & No Prior \\
\hline$\Delta m_{31}^{2}$ & No prior \\
\hline$\theta_{13}$ & $\theta_{13} \pm 0.13^{\circ}$ \\
\hline$\theta_{23}$ & No prior \\
\hline
\end{tabular}

Table 3. List of fitted values and relative gaussian priors considered in this analysis. $\theta_{13}$ refers to the values listed in table 2 .

squared distribution. Specifically, the negative log-likelihood function is defined as:

$$
\begin{aligned}
\chi^{2}= & -2 \log L=\chi_{\text {stat }}^{2}+\chi_{\text {syst }}^{2} \\
= & 2 \sum_{i=1}^{N_{E^{\prime}}} \sum_{j=1}^{N_{\text {cos } \theta^{\prime}}} \sum_{t=1}^{3}\left[N_{i j t}^{\text {model }}(\eta)-N_{i j t}^{\text {data }}+N_{i j t}^{\text {data }} \log \left(\frac{N_{\mathrm{ijt}}^{\text {data }}}{N_{i j t}^{\text {model }}(\eta)}\right)\right] \\
& +\sum_{k=1}^{N_{\text {Syst }}}\left(\frac{\eta_{k}^{\prime}-\left\langle\eta_{k}^{\prime}\right\rangle}{\sigma_{\eta_{k}^{\prime}}}\right)^{2},
\end{aligned}
$$

where $N_{i j t}^{\text {model }}$ and $N_{i j t}^{\text {data }}$ represent the number of expected and measured events in bin $(i, j)$ respectively and the sum over $t$ runs over the three event topologies: tracks, intermediates and showers. $\eta$ represents the model parameters, which comprise both the oscillation parameters listed in table 2 , and nuisance parameters $\eta^{\prime}$, which are related to systematic uncertainties. The second sum runs over the nuisance parameters and $\left\langle\eta_{k}^{\prime}\right\rangle$ is the assumed prior of the parameter $k$ and $\sigma_{\eta_{k}^{\prime}}$ its uncertainty. The set of free parameters considered in this analysis, together with the assumed gaussian priors with mean $\mu$ and standard deviation $\sigma$, is summarised in table 3. Where the uncertainties on the neutrino flux are taken from ref. [41] and the uncertainty on the detector energy scale follows the investigations reported in ref. [2] (section 3.4.6). Specifically:

1. the ratio between the total number of $\nu_{e}$ and $\bar{\nu}_{e}$ is allowed to vary with a standard deviation of $7 \%$ of the parameter's nominal value, 
2. the ratio between the total number of $\nu_{\mu}$ and $\bar{\nu}_{\mu}$ is allowed to vary with a standard deviation of $5 \%$ of the parameter's nominal value,

3. the ratio between the total number of $\nu_{e}$ and $\nu_{\mu}$ is allowed to vary with a standard deviation of $2 \%$ of the parameter's nominal value,

4. the number of $\mathrm{NC}$ events is scaled by the $N C$ scale factor, to which no constraint is applied,

5. the absolute energy scale of the detector, which depends on the knowledge of the PMT efficiencies and the water optical properties, as discussed in ref. [22], is allowed to vary with a standard deviation of $5 \%$ around its nominal value,

6. the energy slope of the neutrino flux energy distribution is allowed to vary without constraint,

7. the ratio of upgoing to horizontally-going neutrinos, the zenith angle slope, is allowed to vary with a standard deviation of $2 \%$ of the parameter's nominal value,

8. the number of events in the three classes is allowed to vary without constraints,

9. $\Delta m_{31}^{2}$ and $\theta_{23}$ are allowed to vary without constraints,

10. $\theta_{13}$ is allowed to vary within a $1 \sigma$ window of the parameter's nominal value, which corresponds to $0.13^{\circ}$ for both NO and IO.

In the following section, the ORCA sensitivity to the active-sterile parameters is presented.

\section{Sensitivity results}

The ORCA sensitivity to the active-sterile mixing angles is here presented. The Asimov dataset is obtained using the parameters in table 2, assuming no sterile neutrino in NO and IO. No assumption is made on NMO: the fit is marginalised over NMO. This allows to conservatively take into account degeneracies between NMO and the sterile parameters.

At the SBL neutrino mass scale, $\Delta m_{41}^{2} \sim 1 \mathrm{eV}^{2}$, correlated constraints in the $\theta_{24}-\theta_{34}$ parameter space are obtained. And, for a more general analysis, sensitivities to the mixing elements $\theta_{14}, \theta_{24}, \theta_{\mu e}$ and $\theta_{34}$ over the range $\Delta m_{41}^{2} \in\left[10^{-5}, 10\right] \mathrm{eV}^{2}$ are presented.

\subsection{Sensitivity to $\theta_{24}-\theta_{34}$ in the large $\Delta m_{41}^{2}$ limit}

As shown in figure 4 , in this sterile mass region, the track channel appears to be the most effective in constraining $\theta_{24}$ and $\theta_{34}$.

As stated in section $3, \delta_{24}$ highly impacts the analysis due to matter effects. Therefore, $\delta_{24}$ is kept free in the fit. Whereas, we investigated the impact of $\theta_{14}$ and found it to be negligible, therefore $\theta_{14}$ and $\delta_{14}$ are fixed to zero in this part of the analysis.

Figure 7 shows the $90 \%$ and $99 \%$ C.L. ORCA sensitivity on $\sin ^{2} \theta_{24}$ and $\sin ^{2} \theta_{34} \cos ^{2} \theta_{24}$ for three years of data taking. The ORCA sensitivity is compared to upper limits from 

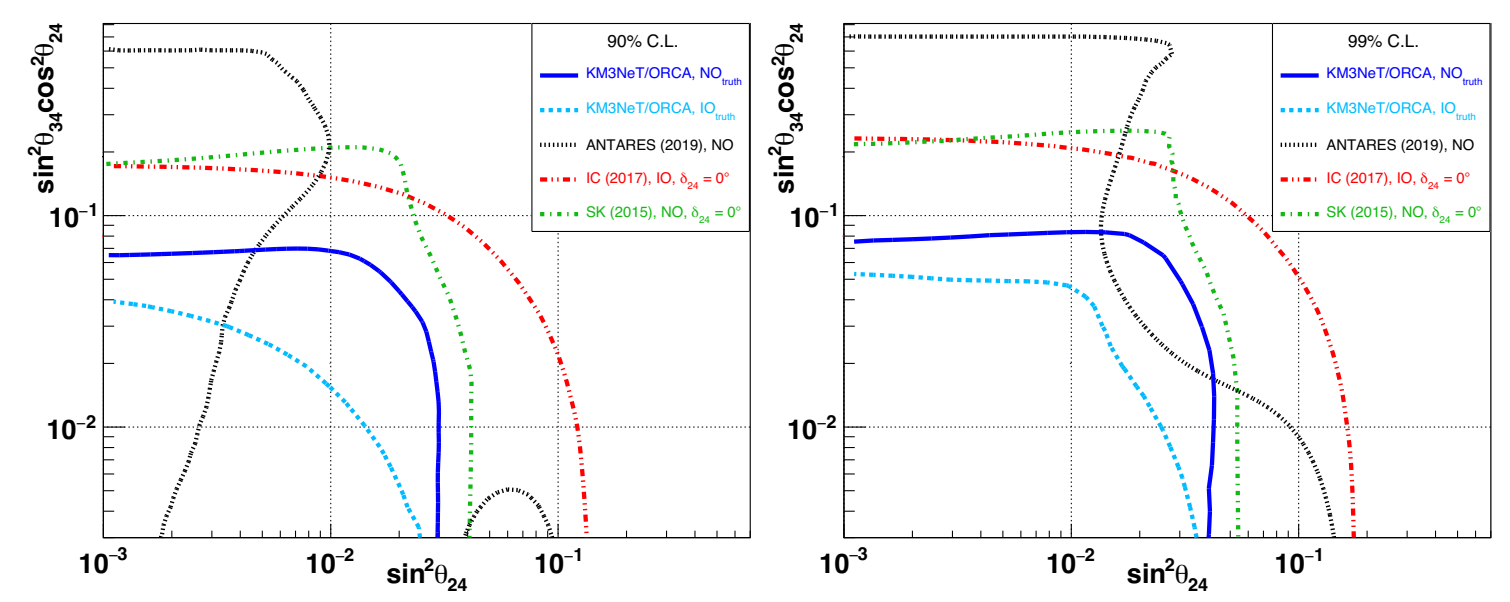

Figure 7. The $90 \%$ (left) and $99 \%$ C.L. (right) KM3NeT/ORCA sensitivity to the mixing parameters $\theta_{24}-\theta_{34}$, with $\Delta m_{41}^{2}=1 \mathrm{eV}^{2}$, for three years of assumed data taking. The obtained sensitivity is compared with current upper limits from ANTARES [38], IceCube/DeepCore (IC) [39] and SK [42]. If not explicitly stated, $\delta_{24}$ is free in the fit: this applies to the results from ORCA and ANTARES. The excluded region is the one on the top right of the lines.

other neutrino experiments, namely ANTARES [38], IceCube/DeepCore [39] and SK [42]. In order to highlight the impact of $\delta_{24}$ in the final constraints, ANTARES has presented upper limits [38] with $\delta_{24}$ fixed to 0 and free. Allowing $\delta_{24}$ to be free worsens the constraints on $\theta_{24}$ and $\theta_{34}$ and it needs to be considered as a free parameter by all the analyses in which Earth matter effects are not negligible. Here, only the analysis with $\delta_{24}$ free is presented. The impact of this quantity in the ORCA sensitivity can be found in ref. [43]: it is maximal when $\sin ^{2} \theta_{24}=\sin ^{2} \theta_{34} \cos ^{2} \theta_{24}$, for which case it worsens the sensitivity by about a factor of two for $\sin ^{2} \theta_{24}$ and a factor three for $\sin ^{2} \theta_{34} \cos ^{2} \theta_{24}$.

Due to the degeneracy driven by NMO and $\delta_{24}$, discussed in section 3 , the ORCA Asimov dataset in NO and $\delta_{24}$ free (blue line) can be directly compared with IceCube/DeepCore IO and $\delta_{24}=0$ (red line). For SK, upper limits with IO are not available, therefore the ones with $\mathrm{NO}$ and $\delta_{24}=0$ are here reported.

From figure 7 it can be concluded that ORCA is competitive in constraining the mixing elements $\theta_{24}$ and $\theta_{34}$, and it is expected to improve the sensitivity to $\sin ^{2} \theta_{34} \cos ^{2} \theta_{24}$ by over a factor of two with respect to current limits.

\subsection{Sensitivity to $\theta_{24}$ for different $\Delta m_{41}^{2}$ values}

Figure 8 shows the $90 \%$ and $99 \%$ C.L. ORCA sensitivity to $\sin ^{2} \theta_{24}$ assuming three years of data taking. For this analysis, $\theta_{14}, \theta_{34}, \delta_{14}$ and $\delta_{24}$ are set free in the fit, since their effects on the results of the analysis are expected to be not negligible.

The ORCA sensitivity is compared with upper limits from cosmology [13] for which only $95 \%$ C.L. are available, and upper limits from MINOS/MINOS+ [44], IceCube [45] and SK [42]. 

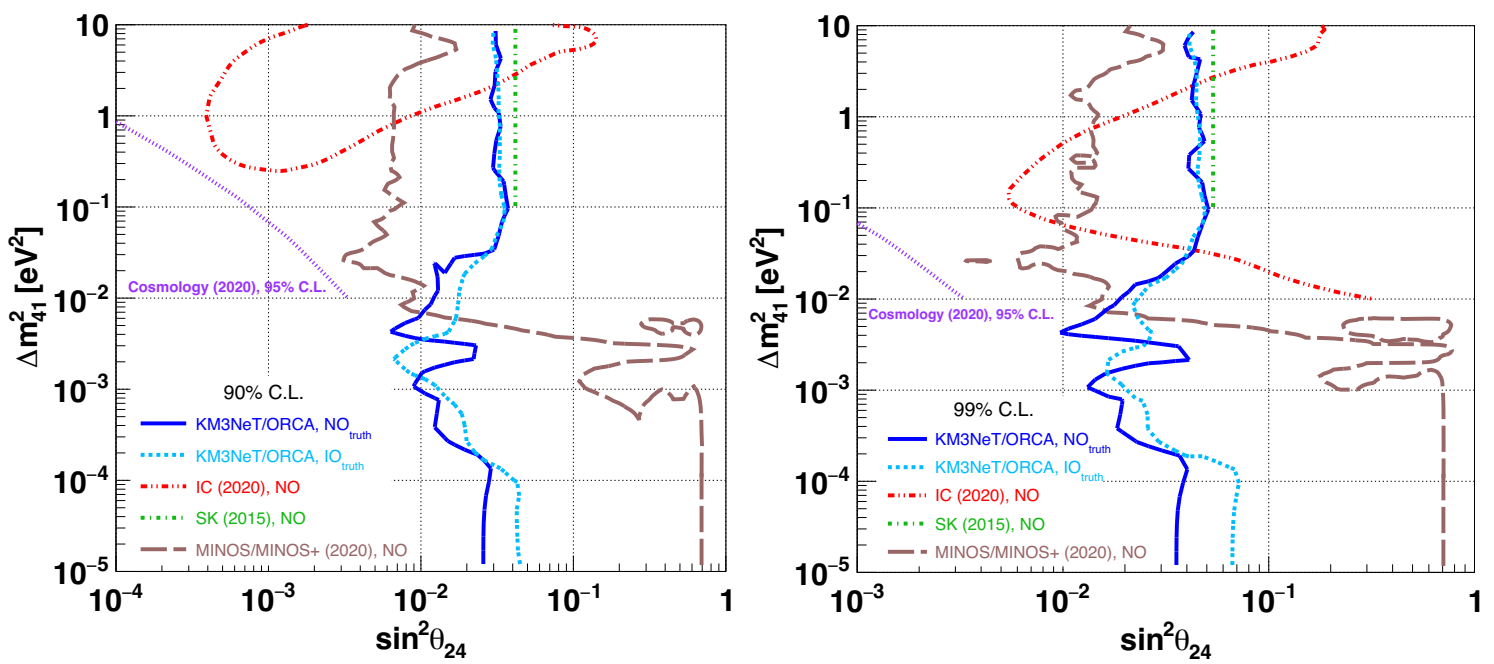

Figure 8. The $90 \%$ (left) and $99 \%$ C.L. (right) KM3NeT/ORCA sensitivity to the mixing parameter $\theta_{24}$, assuming three years of data taking. The obtained sensitivity is compared with current upper limits from cosmology [13], MINOS/MINOS+ [44], IceCube (IC) [45] and SK [42]. The excluded region is the one on the right of the lines, for IceCube at $90 \%$ C.L. it is the external region to the closed contour line.

Both plots show that ORCA is less competitive than MINOS/MINOS+ and IceCube for HF. KM3NeT/ARCA would be better suited to test $\sin ^{2} \theta_{24}$ in this region. In the LF region, ORCA is able to improve current limits on $\sin ^{2} \theta_{24}$ by more than one order of magnitude.

\subsection{Sensitivity to $\theta_{14}$ for different $\Delta m_{41}^{2}$ values}

Figure 9 shows the $95 \%$ C.L. ORCA sensitivity to $\sin ^{2} \theta_{14}$ after three years of data taking. The choice to show the sensitivity at such a level of confidence is motivated by the goal to have a fair comparison with the other experiments, for which the majority of the available upper limits and sensitivity is reported at $95 \%$ C.L. For this analysis, $\theta_{24}, \theta_{34}, \delta_{14}$ and $\delta_{24}$ are free in the fit, since their effects on the results of the analysis are expected to be not negligible. Figure 5 shows that, in the HF region, shower-like events are the most affected by $\theta_{14}$ and in the optimal energy region for ORCA $\left(E^{\prime}<10 \mathrm{GeV}\right)$. However, they are concentrated in the nearly-horizontal region $\left(-0.1<\cos \theta_{Z}<-0.6\right)$. Nevertheless, ORCA has a competitive sensitivity to Daya Bay+Bugey-3 [44] and STEREO [46] in the HF region. Moreover, ORCA will also be able to test part of the Neutrino-4 allowed region [47]. On the contrary, the global fit regions can not be reached with three years of data taking.

\subsection{Sensitivity to $\left|U_{\mu e}\right|^{2}$ for different $\Delta m_{41}^{2}$ values}

Since ORCA can observe both $\nu_{e}$ and $\nu_{\mu}$ disappearance, the effective mixing element $\left|U_{\mu e}\right|^{2}=\sin ^{2} 2 \theta_{\mu e}=4\left|U_{e 4}\right|^{2}\left|U_{\mu 4}\right|^{2}$ can be constrained directly. In this case, $\theta_{14}$ and $\theta_{24}$ are left free in the fit, however, their combination is constrained to match the appropriate $\theta_{\mu e}$ value by introducing a penalty term in the likelihood with a very small prior uncertainty 


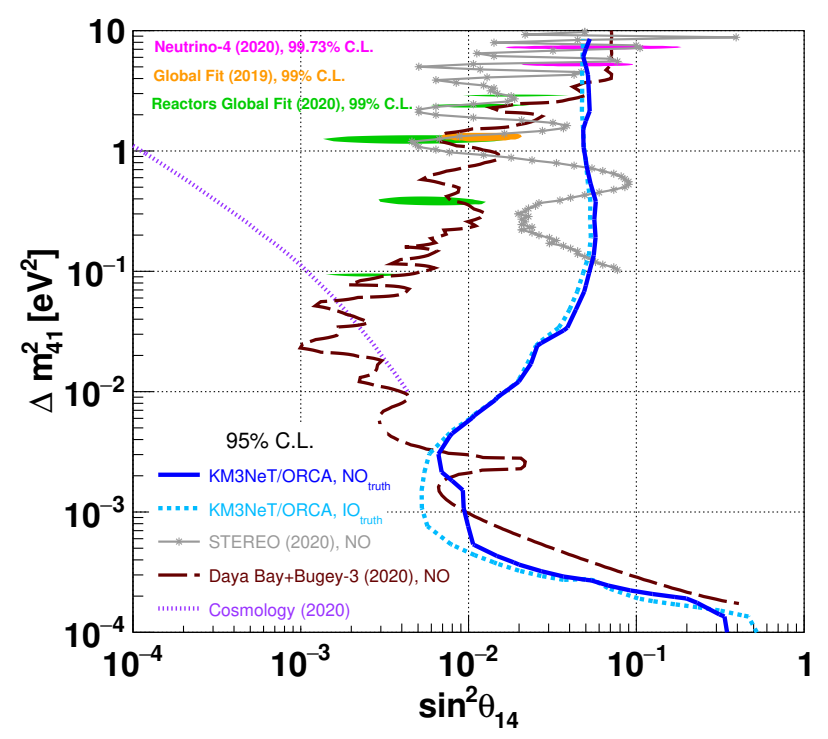

Figure 9. The $95 \%$ C.L. KM3NeT/ORCA sensitivity to the mixing parameter $\theta_{14}$, for different values of $\Delta m_{41}^{2}$, for three years of data taking. Sensitivity results are compared with current upper limits from cosmology [13], STEREO [46], and Daya Bay+Bugey-3 [44]. Current anomaly regions are also reported, from Neutrino-4 [47], global fits [9] and reactors global fits [48]. The excluded region is the one on the right of the lines.

of $10^{-6}$. Figure 10 shows the $90 \%$ and $99 \%$ C.L. ORCA sensitivity to $\left|U_{\mu e}\right|^{2}$, compared with current upper limits from Daya Bay+Bugey-3+MINOS/MINOS+ [44], KARMEN [49], and NOMAD [50].

Figure 10 shows that, after three years of data taking, ORCA will be able to test the majority of the LSND [11] and MiniBoone [12] anomaly region. Moreover, current limits on $\sin ^{2} 2 \theta_{\mu e}$ will be improved by 1-2 orders of magnitude in the LF region.

\subsection{Sensitivity to $\theta_{34}$ for different $\Delta m_{41}^{2}$ values}

Figure 11 shows the ORCA sensitivity at $99 \%$ C.L. to $\sin ^{2} \theta_{34}$ after three years of data taking. Here, $\theta_{14}, \theta_{24}, \delta_{14}$ and $\delta_{24}$ are set free in the fit. Upper limits from cosmology [13], IceCube/DeepCore [39] and SK [42] are also reported. In the LF region there are no upper limits on $\theta_{34}$ coming from other experiments.

ORCA is able to constrain $\theta_{34}$ over a broad range of $\Delta m_{41}^{2}$. In the HF region, consistently with figure 7 , ORCA can improve current upper limits on $\sin ^{2} \theta_{34}$ by about a factor two.

\section{Summary and conclusions}

KM3NeT/ORCA, a neutrino detector under construction in the Mediterranean Sea, is optimised for oscillation studies with atmospheric neutrinos in the $\mathrm{GeV}$ energy range. In this paper, it has been shown that the ORCA detector has a great potential to search for the presence of a light sterile neutrino in the range $\Delta m_{41}^{2} \in\left[10^{-5}, 10\right] \mathrm{eV}^{2}$, by fitting the expected number of observed events classified in three topologies, namely track, intermediate 

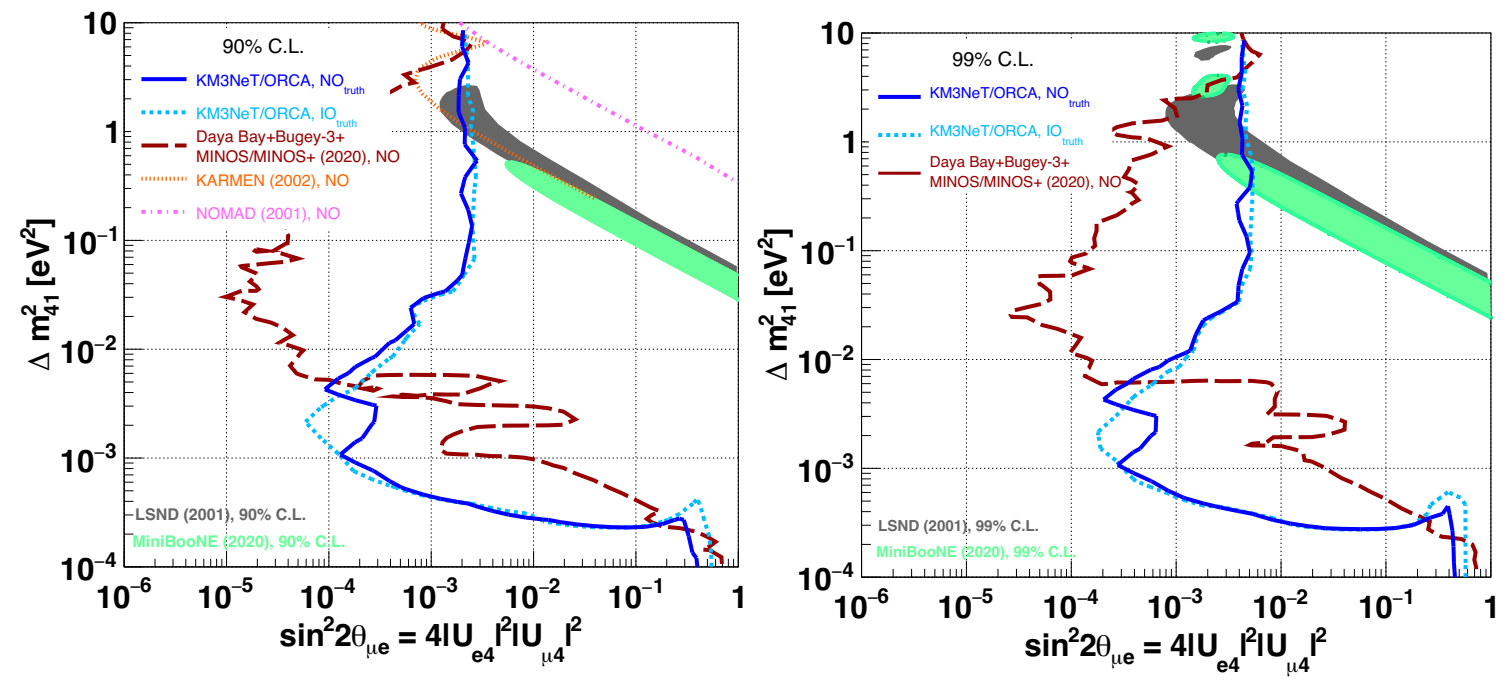

Figure 10. The $90 \%$ (left) and 99\% C.L. (right) KM3NeT/ORCA sensitivity to the mixing parameter $\left|U_{\mu e}\right|^{2}$, assuming three years of data taking. Sensitivity results are compared with current upper limits from Daya Bay+Bugey-3+MINOS/MINOS+[44], KARMEN [49] and NOMAD [50]. Current anomaly regions from LSND [11] and MiniBooNE [12] are also reported. The excluded region is the one on the right of the lines.

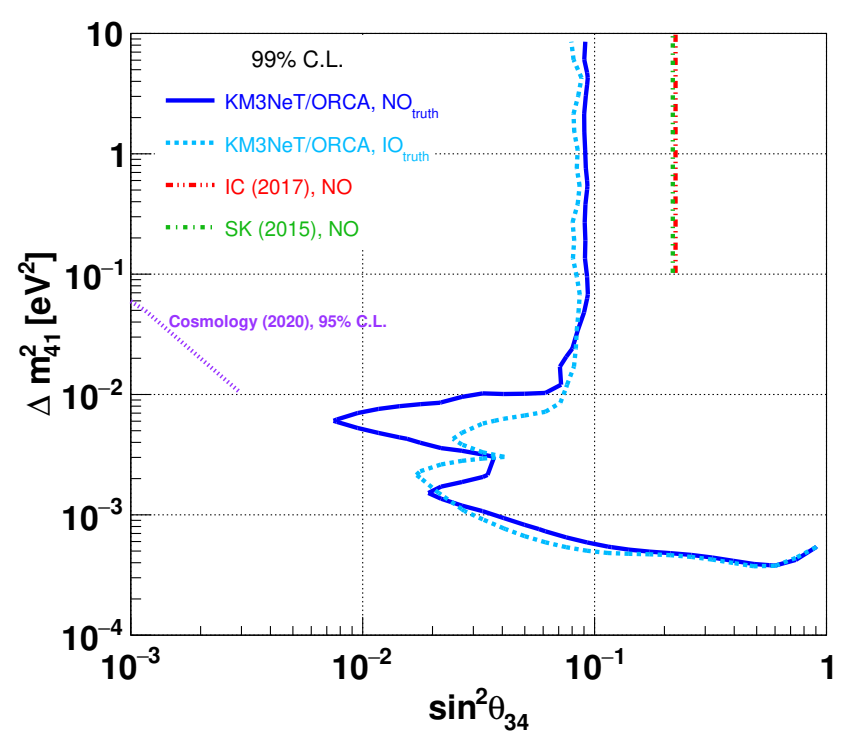

Figure 11. The $99 \%$ C.L. KM3NeT/ORCA sensitivity to the mixing parameter $\theta_{34}$, for different values of $\Delta m_{41}^{2}$, for three years of data taking. Sensitivity results are compared with current upper limits from cosmology [13], IceCube/DeepCore [39] and SK [42]. The excluded region is the one on the right of the lines. 
and shower events. With this methodology, ORCA can probe regions in the active-sterile mixing elements $\theta_{14}, \theta_{24}, \theta_{34}$ and the effective parameter $\theta_{\mu e}$, not yet constrained by current experiments. Particularly, after three years of data taking, ORCA can improve current limits on $\sin ^{2} \theta_{34} \cos ^{2} \theta_{24}$ by about a factor of two, in case of null result, for an eV-mass sterile neutrino. For lower sterile neutrino masses, down to $\Delta m_{41}^{2} \rightarrow 10^{-5} \mathrm{eV}^{2}$, ORCA will be able to test the unexplored region of the $\sin ^{2} \theta_{24}$ parameter, and $\sin ^{2} 2 \theta_{\mu e}$ effective parameter space down to about two orders of magnitude with respect to current limits. The ORCA sensitivity to $\sin ^{2} \theta_{14}$ is comparable to current upper limits. Finally, in case of null result, ORCA will able to improve current limits on $\sin ^{2} \theta_{34}$ by about a factor two for an $\mathrm{eV}$-mass sterile neutrino, and it is the first experiment, to date, able to constrain $\theta_{34}$ in the very low sterile mass region.

\section{Acknowledgments}

The authors acknowledge the financial support of the funding agencies: Agence Nationale de la Recherche (contract ANR-15-CE31-0020), Centre National de la Recherche Scientifique (CNRS), Commission Européenne (FEDER fund and Marie Curie Program), Institut Universitaire de France (IUF), LabEx UnivEarthS (ANR-10-LABX-0023 and ANR18-IDEX-0001), Paris Île-de-France Region, France; Shota Rustaveli National Science Foundation of Georgia (SRNSFG, FR-18-1268), Georgia; Deutsche Forschungsgemeinschaft (DFG), Germany; The General Secretariat of Research and Technology (GSRT), Greece; Istituto Nazionale di Fisica Nucleare (INFN), Ministero dell'Università e della Ricerca (MIUR), PRIN 2017 program (Grant NAT-NET 2017W4HA7S) Italy; Ministry of Higher Education Scientific Research and Professional Training, ICTP through Grant AF-13, Morocco; Nederlandse organisatie voor Wetenschappelijk Onderzoek (NWO), the Netherlands; The National Science Centre, Poland (2015/18/E/ST2/00758); National Authority for Scientific Research (ANCS), Romania; Ministerio de Ciencia, Innovación, Investigación y Universidades (MCIU): Programa Estatal de Generación de Conocimiento (refs. PGC2018-096663-B-C41, -A-C42, -B-C43, -B-C44) (MCIU/FEDER), Generalitat Valenciana: Prometeo (PROMETEO/2020/019), Grisolía (ref. GRISOLIA/2018/119) and GenT (refs. CIDEGENT/2018/034, /2019/043, /2020/049) programs, Junta de Andalucía (ref. A-FQM-053-UGR18), La Caixa Foundation (ref. LCF/BQ/IN17/11620019), EU: MSC program (ref. 101025085), Spain.

Open Access. This article is distributed under the terms of the Creative Commons Attribution License (CC-BY 4.0), which permits any use, distribution and reproduction in any medium, provided the original author(s) and source are credited.

\section{References}

[1] V.N. Gribov and B. Pontecorvo, Neutrino astronomy and lepton charge, Phys. Lett. B 28 (1969) 493 [INSPIRE].

[2] KM3Net collaboration, Letter of intent for KM3NeT 2.0, J. Phys. G 43 (2016) 084001 [arXiv: 1601.07459$]$ [INSPIRE]. 
[3] P.A. Machado, O. Palamara and D.W. Schmitz, The short-baseline neutrino program at Fermilab, Ann. Rev. Nucl. Part. Sci. 69 (2019) 363 [arXiv:1903.04608] [INSPIRE].

[4] DUNE collaboration, Deep Underground Neutrino Experiment (DUNE), far detector technical design report, volume I introduction to DUNE, 2020 JINST 15 T08008 [arXiv: 2002.02967] [INSPIRE].

[5] JUNO collaboration, The JUNO experiment, Nuovo Cim. C 39 (2017) 318 [inSPIRE].

[6] K. Abe et al., Letter of intent: the hyper-Kamiokande experiment - detector design and physics potential, arXiv:1109.3262 [INSPIRE].

[7] IceCube-Gen2 collaboration, IceCube-Gen2: the window to the extreme universe, J. Phys. G 48 (2021) 060501 [arXiv: 2008. 04323] [INSPIRE].

[8] ICAL collaboration, Physics potential of the ICAL detector at the India-based Neutrino Observatory (INO), Pramana 88 (2017) 79 [arXiv: 1505.07380] [INSPIRE].

[9] A. Diaz, C.A. Argüelles, G.H. Collin, J.M. Conrad and M.H. Shaevitz, Where are we with light sterile neutrinos?, Phys. Rept. 884 (2020) 1 [arXiv:1906.00045] [INSPIRE].

[10] ALEPH, DELPHI, L3, OPAL, SLD, LEP Electroweak Working Group, SLD Electroweak Group and SLD Heavy Flavour Group collaborations, Precision electroweak measurements on the $Z$ resonance, Phys. Rept. 427 (2006) 257 [hep-ex/0509008] [INSPIRE].

[11] LSND collaboration, Candidate events in a search for $\bar{\nu}_{\mu} \rightarrow \bar{\nu}_{e}$ oscillations, Phys. Rev. Lett. 75 (1995) 2650 [nucl-ex/9504002] [INSPIRE].

[12] MiniBooNE collaboration, Updated MiniBooNE neutrino oscillation results with increased data and new background studies, Phys. Rev. D 103 (2021) 052002 [arXiv:2006.16883] [INSPIRE].

[13] S. Hagstotz et al., Bounds on light sterile neutrino mass and mixing from cosmology and laboratory searches, arXiv: 2003.02289 [INSPIRE].

[14] A.D. Dolgov, Neutrinos and big bang nucleosynthesis, Nuovo Cim. B 117 (2003) 1081 [hep-ph/0203164] [INSPIRE].

[15] S. Gariazzo, Light sterile neutrinos in cosmology, in $17^{\text {th }}$ Lomonosov conference on elementary particle physics, World Scientific, Singapore (2017), pg. 469 [arXiv:1601.01475] [INSPIRE].

[16] J. Lesgourgues and S. Pastor, Massive neutrinos and cosmology, Phys. Rept. 429 (2006) 307 [astro-ph/0603494] [INSPIRE].

[17] Planck collaboration, Planck 2018 results. VI. Cosmological parameters, Astron. Astrophys. 641 (2020) A6 [Erratum ibid. 652 (2021) C4] [arXiv: 1807.06209] [INSPIRE].

[18] M. Archidiacono et al., Pseudoscalar-sterile neutrino interactions: reconciling the cosmos with neutrino oscillations, JCAP 08 (2016) 067 [arXiv: 1606.07673] [INSPIRE].

[19] S. Razzaque and A.Y. Smirnov, Searching for sterile neutrinos in ice, JHEP 07 (2011) 084 [arXiv: 1104.1390] [INSPIRE].

[20] S. Razzaque and A.Y. Smirnov, Searches for sterile neutrinos with IceCube DeepCore, Phys. Rev. D 85 (2012) 093010 [arXiv: 1203.5406] [INSPIRE].

[21] ANTARES collaboration, ANTARES: the first undersea neutrino telescope, Nucl. Instrum. Meth. A 656 (2011) 11 [arXiv:1104.1607] [INSPIRE]. 
[22] KM3NET collaboration, Determining the neutrino mass ordering and oscillation parameters with KM3NeT/ORCA, arXiv:2103.09885 [INSPIRE].

[23] J.A.B. Coelho, OscProb, https://github.com/joaoabcoelho/OscProb.

[24] M. Maltoni and T. Schwetz, Sterile neutrino oscillations after first MiniBooNE results, Phys. Rev. D 76 (2007) 093005 [arXiv: 0705. 0107] [INSPIRE].

[25] KM3NET collaboration, gSeaGen: the KM3NeT GENIE-based code for neutrino telescopes, Comput. Phys. Commun. 256 (2020) 107477 [arXiv: 2003.14040] [INSPIRE].

[26] C. Andreopoulos et al., The GENIE neutrino Monte Carlo generator, Nucl. Instrum. Meth. A 614 (2010) 87 [arXiv: 0905.2517] [INSPIRE].

[27] A.G. Tsirigotis, A. Leisos and S.E. Tzamarias, HOU Reconstruction \& Simulation (HOURS): a complete simulation and reconstruction package for very large volume underwater neutrino telescopes, Nucl. Instrum. Meth. A 626-627 (2011) S185 [inSPIRE].

[28] GEANT4 collaboration, GEANT4 - a simulation toolkit, Nucl. Instrum. Meth. A 506 (2003) 250 [INSPIRE].

[29] M. Honda, M. Sajjad Athar, T. Kajita, K. Kasahara and S. Midorikawa, Atmospheric neutrino flux calculation using the NRLMSISE-00 atmospheric model, Phys. Rev. D 92 (2015) 023004 [arXiv: 1502.03916] [INSPIRE].

[30] Y. Becherini, A. Margiotta, M. Sioli and M. Spurio, A parameterisation of single and multiple muons in the deep water or ice, Astropart. Phys. 25 (2006) 1 [hep-ph/0507228] [InSPIRE].

[31] G. Carminati, A. Margiotta and M. Spurio, Atmospheric MUons from PArametric formulas: a fast GEnerator for neutrino telescopes (MUPAGE), Comput. Phys. Commun. 179 (2008) 915 [arXiv: 0802.0562] [INSPIRE].

[32] ANTARES collaboration, Monte Carlo simulations for the ANTARES underwater neutrino telescope, JCAP 01 (2021) 064 [arXiv:2010.06621] [INSPIRE].

[33] S. Bourret, Neutrino oscillations and earth tomography with KM3NeT-ORCA, Ph.D. thesis, APC, Paris, France (2018).

[34] S. Adrián-Martínez et al., Intrinsic limits on resolutions in muon- and electron-neutrino charged-current events in the KM3NeT/ORCA detector, JHEP 05 (2017) 008 [arXiv: 1612.05621] [INSPIRE].

[35] R.J. Barlow and C. Beeston, Fitting using finite Monte Carlo samples, Comput. Phys. Commun. 77 (1993) 219 [INSPIRE].

[36] I. Esteban, M.C. Gonzalez-Garcia, A. Hernandez-Cabezudo, M. Maltoni and T. Schwetz, Global analysis of three-flavour neutrino oscillations: synergies and tensions in the determination of $\theta_{23}, \delta_{\mathrm{CP}}$, and the mass ordering, JHEP 01 (2019) 106 [arXiv:1811.05487] [INSPIRE].

[37] A.M. Dziewonski and D.L. Anderson, Preliminary reference earth model, Phys. Earth Plan. Int. 25 (1981) 297.

[38] ANTARES collaboration, Measuring the atmospheric neutrino oscillation parameters and constraining the $3+1$ neutrino model with ten years of ANTARES data, JHEP 06 (2019) 113 [arXiv: 1812.08650] [INSPIRE].

[39] ICECuBE collaboration, Search for sterile neutrino mixing using three years of IceCube DeepCore data, Phys. Rev. D 95 (2017) 112002 [arXiv:1702.05160] [INSPIRE]. 
[40] G. Cowan, K. Cranmer, E. Gross and O. Vitells, Asymptotic formulae for likelihood-based tests of new physics, Eur. Phys. J. C 71 (2011) 1554 [Erratum ibid. 73 (2013) 2501] [arXiv: 1007.1727] [INSPIRE].

[41] G.D. Barr, T.K. Gaisser, S. Robbins and T. Stanev, Uncertainties in atmospheric neutrino fluxes, Phys. Rev. D 74 (2006) 094009 [astro-ph/0611266] [InSPIRE].

[42] SUPER-KAmIOKANDE collaboration, Limits on sterile neutrino mixing using atmospheric neutrinos in Super-Kamiokande, Phys. Rev. D 91 (2015) 052019 [arXiv:1410.2008] [INSPIRE].

[43] KM3NET, ANTARES collaboration, Search for sterile neutrinos with KM3NeT/ORCA, PoS ICRC2019 (2021) 870 [INSPIRE].

[44] MINOS+ and DAYA BAY collaborations, Improved constraints on sterile neutrino mixing from disappearance searches in the MINOS, MINOS+, Daya Bay, and Bugey-3 experiments, Phys. Rev. Lett. 125 (2020) 071801 [arXiv: 2002.00301] [INSPIRE].

[45] ICECuBE collaboration, Searching for eV-scale sterile neutrinos with eight years of atmospheric neutrinos at the IceCube neutrino telescope, Phys. Rev. D 102 (2020) 052009 [arXiv: 2005.12943] [INSPIRE].

[46] STEREO collaboration, Improved sterile neutrino constraints from the STEREO experiment with 179 days of reactor-on data, Phys. Rev. D 102 (2020) 052002 [arXiv:1912.06582] [INSPIRE].

[47] A.P. Serebrov et al., Search for sterile neutrinos with the neutrino-4 experiment and measurement results, Phys. Rev. D 104 (2021) 032003 [arXiv:2005.05301] [InSPIRE].

[48] J.M. Berryman and P. Huber, Sterile neutrinos and the global reactor antineutrino dataset, JHEP 01 (2021) 167 [arXiv:2005.01756] [INSPIRE].

[49] B. Armbruster et al., KARMEN limits on $\nu_{e} \rightarrow \nu_{\tau}$ oscillations in two neutrino and three neutrino mixing schemes, Phys. Rev. C 57 (1998) 3414 [hep-ex/9801007] [INSPIRE].

[50] NOMAD collaboration, Search for $\nu_{\mu} \rightarrow \nu_{e}$ oscillations in the NOMAD experiment, Phys. Lett. B $5 \mathbf{7 0}$ (2003) 19 [hep-ex/0306037] [InSPIRE]. 